JOURNAL OF THE

AMERICAN MATHEMATICAL SOCIETY

Volume 25, Number 3, July 2012, Pages 715-738

S $0894-0347(2012) 00729-2$

Article electronically published on January 27, 2012

\title{
p-ADIC PERIODS AND DERIVED DE RHAM COHOMOLOGY
}

\author{
A. BEILINSON
}

To Irene

\section{INTRODUCTION}

For a smooth variety $X$ over a base field of characteristic 0 we have its algebraic de Rham cohomology $H_{\mathrm{dR}}(X):=H^{\cdot}\left(X_{\mathrm{Zar}}, \Omega_{X}\right)$; for nonsmooth $X$, one defines $H_{\mathrm{dR}}(X)$ using cohomological descent as in Deligne [D]. If the base field is $\mathbb{C}$, then one has the Betti cohomology $H_{\mathrm{B}}(X):=H^{\cdot}\left(X_{\mathrm{cl}}, \mathbb{Q}\right)$ and a canonical period isomorphism ("integration of algebraic differential forms over topological cycles")

$$
\rho: H_{\mathrm{dR}}(X) \stackrel{\sim}{\rightarrow} H_{\mathrm{B}}(X) \otimes \mathbb{C}
$$

compatible with the $\operatorname{Gal}(\mathbb{C} / \mathbb{R})$-conjugation. To define $\rho$, consider the analytic de Rham cohomology $H_{\mathrm{dR}}\left(X_{\mathrm{an}}\right)$. There are evident maps

$$
H_{\mathrm{dR}}(X) \stackrel{\alpha}{\rightarrow} H_{\mathrm{dR}}^{\cdot}\left(X_{\mathrm{an}}\right) \stackrel{\beta}{\leftarrow} H_{\mathrm{B}}(X) \otimes \mathbb{C} .
$$

Then $\beta$ is an isomorphism due to the Poincaré lemma, and $\rho:=\beta^{-1} \alpha$ (the fact that $\rho$ is an isomorphism was established by Grothendieck $[\mathrm{Gr}$ ).

Suppose our base field is an algebraic closure $\bar{K}$ of a $p$-adic field $K$ (say, $K=\mathbb{Q}_{p}$ ). The role of $H_{\mathrm{B}}(X)$ is now played by the $p$-adic étale cohomology $H_{\text {ett }}\left(X, \mathbb{Q}_{p}\right)$, and Fontaine conjectured in $[\mathrm{F} 1] 1$ the existence of a natural $p$-adic period isomorphism

$$
\rho: H_{\mathrm{dR}}(X) \otimes_{\bar{K}} \mathrm{~B}_{\mathrm{dR}} \stackrel{\sim}{\rightarrow} H_{\dot{\mathrm{et}}}\left(X, \mathbb{Q}_{p}\right) \otimes \mathrm{B}_{\mathrm{dR}} .
$$

Here $\mathrm{B}_{\mathrm{dR}}$ is Fontaine's $p$-adic periods field ([F1, F3]). Recall that it is a complete discretely-valued field whose ring of integers $\mathrm{B}_{\mathrm{dR}}^{+}$contains $\bar{K}$, the residue field $\mathrm{B}_{\mathrm{dR}}^{+} / \mathfrak{m}_{\mathrm{dR}}$ is Tate's field $\mathbb{C}_{p}$, the cotangent line $\mathfrak{m}_{\mathrm{dR}} / \mathfrak{m}_{\mathrm{dR}}^{2}$ is the Tate twist $\mathbb{C}_{p}(1)$. Both sides of (0.3) carry natural filtrations (coming from the filtration of $\mathrm{B}_{\mathrm{dR}}$ by powers of $\mathfrak{m}_{\mathrm{dR}}$ and the Hodge-Deligne filtration on $\left.H_{\mathrm{dR}}(X)\right)$, and $\rho$ is compatible with them and with the $\operatorname{Gal}(\bar{K} / K)$-conjugation. Moreover, as was envisioned by Fontaine and Jannsen ( $[\mathrm{F} 4, \mathrm{~J}]$ ), the matrix coefficients of $\rho$ lie in the subring $\bar{K} \mathrm{~B}_{\mathrm{st}}$ of $\mathrm{B}_{\mathrm{dR}} 2^{2}$ and $\rho$ is compatible with the extra symmetries of log crystalline cohomology.

Received by the editors February 22, 2011 and, in revised form, November 16, 2011, and January 5, 2012.

2010 Mathematics Subject Classification. Primary 14F30, 14F40; Secondary 14F20.

Key words and phrases. p-Adic periods, derived de Rham cohomology, h-topology, alterations.

The author was supported in part by NSF grant DMS-1001660.

${ }^{1}$ The assumption of loc. cit. that $X$ is proper and smooth is redundant.

${ }^{2}$ This assertion is true for a general reason, since, by Berger's theorem $\overline{B e r}$, de Rham Galois modules are potentially log crystalline.

(C)2012 American Mathematical Society Reverts to public domain 28 years from publication 
The $p$-adic period map was defined in three different ways in works of, respectively, Faltings, Niziol, and Tsuji (with prior crucial input of Bloch, Fontaine, Hyodo, Kato, Kurihara, and Messing; the nonproper setting was treated by Yamashita [Y]), see [Fa1], [Fa2], [N1], [N2], Ts1], Ts2]; the three $\rho$ 's coincide by [N3].

In the article we give another construction of $\rho$ which is fairly direct and has the same flavor as the classical picture (0.2). The tools are derived de Rham cohomology of Illusie [Ill2, Ch. VIII] and de Jong's alterations. The companion paper [B] treats the Fontaine-Jannsen side of the story; another approach was developed by Bhatt Bh2. It would be very interesting to see if these methods can help to understand the Riemann-Hilbert correspondence in the $p$-adic setting.

An outline of the construction: First we realize $\mathrm{B}_{\mathrm{dR}}^{+}$as the ring of de Rham $p$-adic constants in the sense of derived algebraic geometry. Namely, let $\mathrm{A}_{\mathrm{dR}}$ be the derived de Rham algebra $L \Omega^{\prime} \hat{O}_{\bar{K}} / O_{K}$ completed with respect to the Hodge filtration $F^{*}$; see [Il2, Ch. VIII (2.1.3.3)]. Here $O_{K}, O_{\bar{K}}$ are the rings of integers in $K, \bar{K}$. Now $\mathrm{B}_{\mathrm{dR}}^{+}$identifies canonically with $\mathrm{A}_{\mathrm{dR}} \widehat{\otimes} \mathbb{Q}_{p}$, where $\widehat{\otimes}$ is the derived completed tensor product, so that $\mathfrak{m}_{\mathrm{dR}}^{i} \stackrel{\sim}{\rightarrow} F^{i} \mathrm{~A}_{\mathrm{dR}} \widehat{\otimes} \mathbb{Q}_{p}$. This fact was observed independently by Fargues [Far.

Let $\mathcal{V a r}_{F}$ be the category of varieties over a field $F$, and $\mathcal{V} a r_{F}^{\text {nc }}$ be the category of regular $F$-varieties $U$ equipped with a regular compactification $\bar{U}$ with normal crossings divisor at infinity. As follows from de Jong's theorem dJ1, the forgetful functor $\mathcal{V}_{F}^{\text {nc }} \rightarrow \mathcal{V}_{F} r_{F},(U, \bar{U}) \mapsto U$, makes $\mathcal{V}_{F}^{\text {nc }}$ a base for the h-topology on $\mathcal{V}_{a r_{F}}$, so h-sheaves on $\mathcal{V} r_{F}$ are the same as sheaves on $\mathcal{V} a r_{F}^{\text {nc }}$ for the induced topology. For $F=\bar{K}$ as above, there is a finer category $\mathcal{V}_{\bar{K}}^{\text {ss }}$ of ss-pairs $(V, \bar{V})$, i.e., smooth $\bar{K}$-varieties $V$ equipped with a semi-stable compactification $\bar{V}$ (that includes compactification in the arithmetic direction). Again by de Jong dJ1, $\mathcal{V} a r_{\bar{K}}^{\text {ss }}$ is a base for the h-topology on $\mathcal{V}_{\bar{K}}$.

Consider the presheaf on $\mathcal{V} a r_{K}^{\mathrm{ss}}$ which assigns to $(V, \bar{V})$ the derived de Rham algebra with $\log$ singularities $R \Gamma\left(\bar{V}, L \Omega_{(\hat{V}, \bar{V}) / O_{K}}\right)$ (see Ol]). Its h-sheafification $\mathcal{A}_{\mathrm{dR}}^{\natural}$ is an h-sheaf of filtered dg algebras on $\mathcal{V} a r_{\bar{K}}$ that contains the constant subsheaf $\mathrm{A}_{\mathrm{dR}}$. The key $p$-adic Poincaré lemma says that the map $A_{d R} \otimes^{L} \mathbb{Z} / p^{n} \rightarrow \mathcal{A}_{d R}^{\natural} \otimes^{L}$ $\mathbb{Z} / p^{n}$ is a filtered quasi-isomorphism. It comes from the next assertion: The hsheafification of the presheaf $(V, \bar{V}) \mapsto H^{b}\left(\bar{V}, \Omega_{(V, \bar{V}) / O_{\bar{K}}}^{a}\right)$, where $\Omega_{(V, \bar{V}) / O_{\bar{K}}}^{a}$ is the usual locally free $\mathcal{O}_{\bar{V}}$-module of forms with $\log$ singularities, is an h-sheaf of $\mathbb{Q}$ vector spaces for $(a, b) \neq(0,0)$. The case $a=0$ is essentially theorem 8.0.1 from Bhatt's thesis [Bh1]; the general result is obtained by a similar method (which uses coverings of families of stable curves that come from the multiplication by $p$ isogeny of the generalized Jacobians).

Set $R \Gamma_{\mathrm{dR}}^{\natural}(X):=R \Gamma\left(X_{\mathrm{h}}, \mathcal{A}_{\mathrm{dR}}^{\natural}\right)$; this is the arithmetic de Rham complex of $X$. By the above, $H^{\cdot}\left(R \Gamma_{\mathrm{dR}}^{\natural}(X) \widehat{\otimes} \mathbb{Q}_{p}\right)$ is a $\mathrm{B}_{\mathrm{dR}}^{+}$-algebra. One has a diagram

$$
H_{\mathrm{dR}}(X) \stackrel{\alpha}{\rightarrow} H^{\cdot}\left(R \Gamma_{\mathrm{dR}}^{\natural}(X) \widehat{\otimes} \mathbb{Q}_{p}\right) \stackrel{\beta}{\leftarrow} H_{\dot{\mathrm{et}}}\left(X, \mathbb{Q}_{p}\right) \otimes \mathrm{B}_{\mathrm{dR}}^{+},
$$

where $\alpha$ is the composition $H_{\mathrm{dR}}^{\cdot}(X) \stackrel{\sim}{\rightarrow} H^{\cdot} R \Gamma_{\mathrm{dR}}^{\natural}(X) \otimes \mathbb{Q} \rightarrow H^{\cdot}\left(R \Gamma_{\mathrm{dR}}^{\natural}(X) \widehat{\otimes} \mathbb{Q}_{p}\right)$ and $\beta$ is the $\mathrm{B}_{\mathrm{dR}}^{+}$-linear extension of the evident map (which comes from the embeddings $\mathbb{Z} / p^{n} \rightarrow \mathcal{A}_{\mathrm{dR}}^{\natural} \otimes^{L} \mathbb{Z} / p^{n}$ and the fact that the h-topology is stronger than the étale one). Since the étale and h-cohomology with torsion coefficients coincide, the Poincaré lemma implies that $\beta$ is an isomorphism. Now the $p$-adic period map $\rho$ is 
the $\mathrm{B}_{\mathrm{dR}}$-linear extension of $\beta^{-1} \alpha$. An explicit computation for $X=\mathbb{G}_{m}$ followed by usual tricks of the trade shows that $\rho$ is a filtered isomorphism.

\section{A derived de Rham construction of $B_{\mathrm{dr}}$}

1.1. The derived p-adic completion. Throughout the article we use (not too heavily) $\mathrm{E}_{\infty}$ algebras, for which we refer to, say, $\left.\mathrm{HS}\right] 3$ Recall that $\mathrm{E}_{\infty}$ algebras are dg algebras whose product is commutative and associative up to coherent higher homotopies (more formally, $\mathrm{E}_{\infty}$ algebras are $\mathrm{dg}$ algebras for a resolution of the commutative algebra operad). A key fact: for any commutative (more generally, $\mathrm{E}_{\infty}$ ) cosimplicial dg algebra the corresponding total complex is naturally an $\mathrm{E}_{\infty}$ algebra. Thus the homotopy limit of a diagram of $\mathrm{E}_{\infty}$ algebras is an $\mathrm{E}_{\infty}$ algebra.

For a projective system of complexes of abelian groups $\ldots \stackrel{\phi_{2}}{\longrightarrow} C_{2} \stackrel{\phi_{1}}{\longrightarrow} C_{1}$, one has holim $C_{n}=\mathcal{C}$ one (id $\left.-\phi: \Pi C_{n} \rightarrow \Pi C_{n}\right)[-1]$, where $\phi\left(\left(c_{n}\right)\right)=\left(\phi_{n}\left(c_{n+1}\right)\right)$. There is an embedding $\lim C_{n}=\operatorname{Ker}(\mathrm{id}-\phi) \hookrightarrow$ holim $C_{n}$. If all $\phi_{n}$ 's are surjective, then id $-\phi$ is surjective; hence $\hookrightarrow$ is a quasi-isomorphism. So holim, being an exact functor, is the right derived functor of lim.

If $C$. is a projective system of $\mathrm{dg}$ algebras, then holim $C_{n}$ is naturally a dg algebra (and the above embedding is an embedding of algebras); if the $C_{n}$ are commutative (or, more generally, $\mathrm{E}_{\infty}$ ) algebras, then holim $C_{n}$ is an $\mathrm{E}_{\infty}$ algebra.

Let $p$ be a prime. Consider the projective system of commutative $\mathrm{dg}$ algebras $C_{n}:=\operatorname{Cone}\left(\mathbb{Z} \stackrel{p^{n}}{\longrightarrow} \mathbb{Z}\right)$. It is a resolution of the projective system $\ldots \rightarrow \mathbb{Z} / p^{2} \rightarrow \mathbb{Z} / p$, so $\mathbb{Z}_{p}^{b}:=\operatorname{holim} C_{n}$ is an $\mathrm{E}_{\infty}$ algebra with $H^{0} \mathbb{Z}_{p}^{b}=\mathbb{Z}_{p}$ and acyclic in nonzero degrees. Set $\mathbb{Q}_{p}^{b}:=\mathbb{Z}_{p}^{b} \otimes \mathbb{Q}$. For any complex $F$ of abelian groups set

$$
F \widehat{\otimes} \mathbb{Z}_{p}:=\operatorname{holim}\left(F \otimes C_{n}\right), \quad F \widehat{\otimes} \mathbb{Q}_{p}:=\left(F \widehat{\otimes} \mathbb{Z}_{p}\right) \otimes \mathbb{Q} .
$$

These are $\operatorname{dg} \mathbb{Z}_{p}^{b}$ and $\mathbb{Q}_{p^{b}}^{b}$-modules, so their cohomology groups are $\mathbb{Z}_{p^{-}}$and $\mathbb{Q}_{p^{-}}$ modules, and $F \mapsto F \widehat{\otimes} \mathbb{Z}_{p}, F \widehat{\otimes} \mathbb{Q}_{p}$ are exact functors. If $F$ is an $\left(\mathrm{E}_{\infty}\right)$ dg algebra, then so are $F \widehat{\otimes} \mathbb{Z}_{p}$ and $F \widehat{\otimes} \mathbb{Q}_{p}$.

Remark. One has an evident projective system $F_{p^{n}}[1] \rightarrow F \otimes C_{n} \rightarrow F / p^{n} F$ of exact triangles; applying holim, we get a canonical exact triangle holim $F_{p^{n}}[1] \rightarrow$ $F \widehat{\otimes} \mathbb{Z}_{p} \rightarrow \operatorname{holim}\left(F / p^{n} F\right)$. Let $\hat{F}:=\varliminf_{i m} F / p^{n} F$ be the $p$-adic completion of $F$ and $T_{p} F:=\varliminf_{p^{n}}$ be the Tate module of $F$. By above, we have a quasi-isomorphism $\hat{F} \stackrel{\sim}{\rightarrow} \operatorname{holim}\left(F / p^{n} F\right)$. Thus if $F$ has no $p$-torsion, then $F \widehat{\otimes} \mathbb{Z}_{p} \stackrel{\sim}{\rightarrow} \hat{F}$. Similarly, if all components of $F$ are $p$-divisible, then one has quasi-isomorphisms $T_{p} F \stackrel{\sim}{\rightarrow}$ holim $F_{p^{n}}$ and $T_{p} F[1] \stackrel{\sim}{\rightarrow} F \widehat{\otimes} \mathbb{Z}_{p}$. We see that $\cdot \widehat{\otimes} \mathbb{Z}_{p}$ is the left derived functor of the $p$-adic completion functor and the right derived functor of $T_{p}[1]$.

Example. For a scheme $X$, its étale $\mathbb{Z}_{p^{-}}$and $\mathbb{Q}_{p^{-}}$-cohomology are $R \Gamma_{\text {ét }}\left(X, \mathbb{Z}_{p}\right):=$ $\operatorname{holim} R \Gamma\left(X_{\text {ét }}, \mathbb{Z} / p^{n}\right)=R \Gamma\left(X_{\text {ét }}, \mathbb{Z}\right) \widehat{\otimes} \mathbb{Z}_{p}, R \Gamma_{\text {ét }}\left(X, \mathbb{Q}_{p}\right):=R \Gamma\left(X_{\text {ét }}, \mathbb{Z}\right) \widehat{\otimes} \mathbb{Q}_{p} 4$

1.2. The derived de Rham algebra. For a morphism of commutative rings $A \rightarrow B$ we denote by $\Omega_{B / A}$ the relative de Rham complex of $B$ over $A$. This is a commutative $\operatorname{dg} A$-algebra with components $\Omega_{B / A}^{i}=\Lambda_{B}^{i} \Omega_{B / A}$, where $\Omega_{B / A}$ is the $B$-module of relative Kähler differentials; it carries a ring filtration $F^{n}=\Omega_{B / A}^{\geq n}$.

\footnotetext{
${ }^{3}$ In loc. cit. $\mathrm{E}_{\infty}$ algebras are called "May algebras".

${ }^{4}$ Since $R \Gamma\left(X_{\text {ét }}, \mathbb{Z} / p^{n}\right)=R \Gamma\left(X_{\text {ét }}, \mathbb{Z}\right) \otimes{ }^{L} \mathbb{Z} / p^{n}$.
} 
We will use the $F$-completed version $L \Omega_{B / A}$ of Illusie's derived de Rham algebra defined in [Ill2, Ch. VIII, (2.1.3.3)]. To construct it, consider the canonical simplicial resolution $P .=P_{A}(B)$. of $B$ from [Ill1, Ch. I,(1.5.5.6)]. This is a simplicial commutative $A$-algebra such that each $P_{i}$ is a polynomial $A$-algebra. The de Rham complexes $\Omega_{P . / A}$ form a simplicial filtered commutative dg $A$-algebra, so the corresponding total complex $L \Omega_{B / A}$ is a filtered commutative dg $A$-algebra (see IIl1, Ch. I, 3.1.3]). Now $L \Omega_{B / A}$ is its completion with respect to the filtration $F$. Here "completion" is understood as a mere projective system of quotients modulo $F^{i}$. One has a natural identification $\operatorname{gr}_{F}^{i} L \Omega_{B / A} \stackrel{\sim}{\rightarrow}\left(L \Lambda_{B}^{i}\left(\mathrm{~L}_{B / A}\right)\right)[-i]$ compatible with the product; here $\mathrm{L}_{B / A}:=\Omega_{P . / A} \otimes_{P .} B$ is the relative cotangent complex and $L \Lambda_{B}^{i}$ is the nonabelian left derived functor of the exterior power functor (see Ch. II and Ch. I of [Ill]). For $A$-flat $B$ 's, the construction is compatible with base change. It is compatible with direct limits. If in the above definition we replace $P$. by any simplicial $A$-algebra resolution of $B$ whose terms are polynomial $A$-algebras, then the output is naturally quasi-isomorphic to $L \Omega_{B / A}$.

The next lemma is a particular case of [Ill1, Ch. I, 4.3.2.1(ii)]. For a flat $B$ module $T$ we denote by $B\langle T\rangle^{`}$ its divided powers symmetric algebra.

Lemma. The complex $L \Lambda_{B}^{i}(T[1])$ is acyclic outside degree $-i$. There is a canonical isomorphism of graded $B$-algebras compatible with base change

$$
H^{-\cdot} L \Lambda_{B}(T[1]) \stackrel{\sim}{\rightarrow} B\langle T\rangle .
$$

1.3. Let $K$ be a $p$-adic field, i.e., a complete discretely-valued field of characteristic zero with perfect residue field $k$ of characteristic $p>0, \bar{K}$ be an algebraic closure of $K$, and $O_{K}, O_{\bar{K}}$ be rings of integers in $K, \bar{K}$. Let $K_{0} \subset K$ be the field of fractions of the Witt vectors $W(k)=O_{K_{0}}$, and let $\mathfrak{a}$ be the fractional ideal in $\bar{K}$ generated by $p^{-\frac{1}{p-1}} \mathfrak{D}_{K / K_{0}}^{-1}$, where $\mathfrak{D}_{K / K_{0}}$ is the different. For an $O_{K}$-algebra $B$ we often write $\Omega_{B}:=\Omega_{B / O_{K}}, L \Omega_{B}:=L \Omega_{B} \hat{i} O_{K}, \mathrm{~L}_{B}=\mathrm{L}_{B / O_{K}}$, etc.

The next key result is due to Fontaine [F2, Th 1]; we include a proof for completeness sake. Consider the map $\mu_{p} \subset O_{\bar{K}}^{\times} \stackrel{d \log }{\longrightarrow} \Omega_{O_{\bar{K}}}$ and its $O_{\bar{K}}$-linear extension

$$
\left(\bar{K} / O_{\bar{K}}\right)(1)=O_{\bar{K}} \otimes \mu_{p^{\infty}} \rightarrow \Omega_{O_{\bar{K}}} .
$$

Theorem. One has $L_{O_{\bar{K}}} \stackrel{\sim}{\rightarrow} \Omega_{O_{\bar{K}}}$, and (1.3.1) is surjective with kernel $\left(\mathfrak{a} / O_{\bar{K}}\right)(1)$.

Proof. If $K^{\prime} / K$ is a finite extension, then $O_{K^{\prime}} / O_{K}$ is a complete intersection. So, if $\pi$ is a generator of $O_{K^{\prime}} / O_{K}, f(t)$ its minimal polynomial, then $\mathrm{L}_{O_{K^{\prime}}}$ is the cone of multiplication by $f^{\prime}(\pi)$ endomorphism of $O_{K^{\prime}}$; hence $\mathrm{L}_{O_{K^{\prime}}} \stackrel{\sim}{\rightarrow} \Omega_{O_{K^{\prime}}}$. Passing to the limit, we get the first assertion. Let us prove the second one.

(i) By the above, $\Omega_{O_{K^{\prime}}} \simeq O_{K^{\prime}} / \mathfrak{D}_{K^{\prime} / K}$. If $K^{\prime \prime} / K^{\prime}$ is another finite extension, then the standard exact triangle of the cotangent complexes reduces to a short exact sequence $0 \rightarrow O_{K^{\prime \prime}} \otimes_{O_{K^{\prime}}} \Omega_{O_{K^{\prime}} / O_{K}} \rightarrow \Omega_{O_{K^{\prime \prime}} / O_{K}} \rightarrow \Omega_{O_{K^{\prime \prime}} / O_{K^{\prime}}} \rightarrow 0$.

(ii) Replacing $K^{\prime}, K$ by $K, K_{0}$ and passing to the limit, we get a short exact sequence $0 \rightarrow O_{\bar{K}} \otimes_{O_{K}} \Omega_{O_{K} / O_{K_{0}}} \rightarrow \Omega_{O_{\bar{K}} / O_{K_{0}}} \rightarrow \Omega_{O_{\bar{K}} / O_{K}} \rightarrow 0$. Thus it suffices to prove the theorem for $K=K_{0}$, which we now assume.

(iii) Set $T:=\operatorname{Ker}\left(\left(\bar{K} / O_{\bar{K}}\right)(1) \rightarrow \Omega\right), F:=K\left(\mu_{p}\right)$. The set of $O_{\bar{K}}$-submodules of $\left(\bar{K} / O_{\bar{K}}\right)(1)$ is totally ordered by inclusion. Thus, since $O_{\bar{K}} \otimes_{O_{F}} \Omega_{O_{F}} \subset \Omega_{O_{\bar{K}}}$ is a nonzero $O_{\bar{K}}$-module generated by $d \log \left(\mu_{p}\right)$, one has $T \subset\left(p^{-1} O_{\bar{K}} / O_{\bar{K}}\right)(1)=O_{\bar{K}} \otimes$ $\mu_{p}$. Since $\Omega_{O_{F}}$ is isomorphic to $O_{F} / p^{1-\frac{1}{p-1}} O_{F}$, one has $T=\left(p^{-\frac{1}{p-1}} O_{\bar{K}} / O_{\bar{K}}\right)(1)$. 
(iv) It remains to prove surjectivity of $\left(\bar{K} / O_{\bar{K}}\right)(1) \rightarrow \Omega_{O_{\bar{K}}}$. Let $K^{\prime} \subset \bar{K}$ be any finite extension of $K$; we want to check that $\Omega_{O_{K^{\prime}}} \subset \Omega_{O_{\bar{K}}}$ lies in $O_{\bar{K}} d \log \left(\mu_{p} \infty\right)$. Suppose $p^{n}$ kills $\Omega_{O_{K^{\prime}}}$. Let us show that $\Omega_{O_{K^{\prime}}} \subset O_{\bar{K}} d \log \left(\mu_{p^{n+1}}\right)$. Set $K^{\prime \prime}:=$ $K^{\prime}\left(\mu_{p^{n+1}}\right)$. The set of $O_{K^{\prime \prime}}$-submodules of $\Omega_{O_{K^{\prime \prime}}}$ is totally ordered. Thus, since $p^{n} d \log \left(\mu_{p^{n+1}}\right) \neq 0$ by (iii), $\Omega_{O_{K^{\prime}}}$ lies in $O_{K^{\prime \prime}} d \log \left(\mu_{p^{n+1}}\right)$, q.e.d.

1.4. For a complex $P$ acyclic in degrees $\neq 0$, we often write $P$ instead of $H^{0} P$.

Consider the filtered commutative $\operatorname{dg} O_{K}$-algebra $\mathrm{A}_{\mathrm{dR}}=\mathrm{A}_{\mathrm{dR} \bar{K} / K}:=L \Omega_{O} K_{/ O_{K}}$ and the corresponding filtered $\mathrm{E}_{\infty} O_{K}$-algebra $\mathrm{A}_{\mathrm{dR}} \widehat{\otimes} \mathbb{Z}_{p}$ (see $\S 1.1$ ). Let us describe the graded $O_{\bar{K}}$-algebras gr ${ }_{F} \mathrm{~A}_{\mathrm{dR}}, \operatorname{gr}_{F}^{*} \mathrm{~A}_{\mathrm{dR}} \widehat{\otimes} \mathbb{Z}_{p}$.

Proposition. (i) The complexes $g r_{F}^{i} A_{d R} \widehat{\otimes} \mathbb{Z}_{p}$ are acyclic in nonzero degrees, and there is a canonical isomorphism of graded algebras

$$
\operatorname{gr}_{F} A_{d R} \widehat{\otimes} \mathbb{Z}_{p} \stackrel{\sim}{\rightarrow} \hat{O}_{\bar{K}}\langle\hat{\mathfrak{a}}(1)\rangle .
$$

(ii) One has $g r_{F}^{0} A_{d R}=A_{d R} / F^{1}=O_{\bar{K}}$, and the complexes $g r_{F}^{i} A_{d R}$ for $i>0$ are acyclic in degrees $\neq 1$. There are natural isomorphisms of $O_{\bar{K}}$-modules

$$
\Omega^{\langle i\rangle}:=H^{1} g r_{F}^{i} A_{d R} \stackrel{\sim}{\rightarrow}\left(\bar{K} / i !^{-1} \mathfrak{a}^{i}\right)(i)=\left(\mathbb{Q}_{p} / \mathbb{Z}_{p}\right) \otimes i !^{-1} \hat{\mathfrak{a}}^{i}(i) .
$$

Proof. (i) By the theorem in $\S 1.3$, one has $\mathrm{L}_{O_{\bar{K}} / O_{K}} \stackrel{\sim}{\rightarrow} \Omega_{O_{\bar{K}}} \stackrel{\sim}{\rightarrow}(\bar{K} / \mathfrak{a})(1)=$ $\left(\mathbb{Q}_{p} / \mathbb{Z}_{p}\right) \otimes \mathfrak{a}(1) ;$ hence $\operatorname{gr}_{F}^{i} \mathrm{~A}_{\mathrm{dR}} \stackrel{\sim}{\rightarrow} L \Lambda_{\mathbb{Z}}^{i}\left(\mathbb{Q}_{p} / \mathbb{Z}_{p}\right)[-i] \otimes \mathfrak{a}^{i}(i)$. One has $L \Lambda_{\mathbb{Z}}^{i}\left(\mathbb{Q}_{p} / \mathbb{Z}_{p}\right) \otimes L$ $\left(\mathbb{Z} / p^{n}\right)=L \Lambda_{\mathbb{Z} / p^{n}}^{i}\left(\left(\mathbb{Q}_{p} / \mathbb{Z}_{p}\right) \otimes^{L}\left(\mathbb{Z} / p^{n}\right)\right)=L \Lambda_{\mathbb{Z} / p^{n}}^{i}\left(\left(\mathbb{Z} / p^{n}\right)[1]\right)$, which identifies with $i !^{-1}\left(\mathbb{Z} / p^{n}\right)[i]$ in a way compatible with the product by (1.2.1). Therefore $\operatorname{gr}_{F}^{*} \mathrm{~A}_{\mathrm{dR}} \otimes^{L}$ $\left(\mathbb{Z} / p^{n}\right) \stackrel{\sim}{\rightarrow} \mathbb{Z} / p^{n}\left\langle\left(\mathfrak{a} / p^{n} \mathfrak{a}\right)(1)\right\rangle$, which yields (1.4.1).

(ii) follows from (i) by the next observation (applied to $C=\operatorname{gr}_{F}^{i} \mathrm{~A}_{\mathrm{dR}}$, with (1.4.2) defined by the condition that $T_{p}(1.4 .2)=(1.4 .1)$ ): If a complex $C$ of abelian groups has $p$-torsion cohomology and $H^{\neq 0}\left(C \otimes^{L} \mathbb{Z} / p\right)=0$, then $H^{1} C$ is $p$-divisible and $H^{\neq 1} C=05$

1.5. By 1.4(i), the algebras $\left(\mathrm{A}_{\mathrm{dR}} / F^{i}\right) \widehat{\otimes} \mathbb{Z}_{p}$, hence $\left(\mathrm{A}_{\mathrm{dR}} / F^{i}\right) \widehat{\otimes} \mathbb{Q}_{p}$, are acyclic in nonzero degree. By loc. cit., $\left(\mathrm{A}_{\mathrm{dR}} / F^{i+1}\right) \widehat{\otimes} \mathbb{Q}_{p}$ is an $i$-truncated dvr with residue field $\mathbb{C}_{p}:=\hat{O}_{\bar{K}} \otimes \mathbb{Q}$, so $\mathrm{A}_{\mathrm{dR}} \widehat{\otimes} \mathbb{Q}_{p}:=\underline{\lim }\left(\mathrm{A}_{\mathrm{dR}} / F^{i}\right) \widehat{\otimes} \mathbb{Q}_{p}$ is a dvr. Let $\mathfrak{m}_{\mathrm{dR}}$ be its maximal ideal; (1.4.1) yields a canonical identification $\mathfrak{m}_{\mathrm{dR}} / \mathfrak{m}_{\mathrm{dR}}^{2}=\operatorname{gr}_{F}^{1} \mathrm{~A}_{\mathrm{dR}} \widehat{\otimes} \mathbb{Q}_{p} \stackrel{\sim}{\rightarrow} \mathbb{C}_{p}(1)$.

Proposition. There is a canonical ring isomorphism of filtered rings

$$
u_{\mathbb{Q}}: B_{d R}^{+} \stackrel{\sim}{\rightarrow} A_{d R} \widehat{\otimes} \mathbb{Q}_{p}
$$

Proof. The ring $\left(\mathrm{A}_{\mathrm{dR}} / F^{i+1}\right) \widehat{\otimes} \mathbb{Z}_{p}$ is an infinitesimal $p$-adic $O_{K}$-thickening of $\hat{O}_{K}$ $=\left(\mathrm{A}_{\mathrm{dR}} / F^{1}\right) \widehat{\otimes} \mathbb{Z}_{p}$ of order $\leq i$ (see [F3, 1.1]). Let $\mathrm{A}_{\mathrm{inf}} / F^{i+1}$ be the universal thickening ([F3, 1.3]); we have a canonical map $u_{i}: \mathrm{A}_{\text {inf }} / F^{i+1} \rightarrow\left(\mathrm{A}_{\mathrm{dR}} / F^{i+1}\right) \widehat{\otimes} \mathbb{Z}_{p}$. Since $\mathrm{B}_{\mathrm{dR}}^{+} / F^{i+1}:=\left(\mathrm{A}_{\mathrm{inf}} / F^{i+1}\right) \otimes \mathbb{Q}$ is an $i$-truncated dvr and $u_{1}$ is an isomorphism by [F3, 1.4.3], $u_{i \mathbb{Q}}: \mathrm{B}_{\mathrm{dR}}^{+} / F^{i+1} \stackrel{\sim}{\rightarrow}\left(\mathrm{A}_{\mathrm{dR}} / F^{i+1}\right) \widehat{\otimes} \mathbb{Q}_{p}$. Set $u_{\mathbb{Q}}:=\varliminf_{\varlimsup} u_{i \mathbb{Q}}$.

Remarks. (i) The map $\mathrm{A}_{\mathrm{dR}} \rightarrow \mathrm{A}_{\mathrm{dR}} / F^{1}=O_{\bar{K}}$ yields an isomorphism $\mathrm{A}_{\mathrm{dR}} \otimes \mathbb{Q} \stackrel{\sim}{\rightarrow} \bar{K}$. Thus the morphism $\mathrm{A}_{\mathrm{dR}} \otimes \mathbb{Q} \rightarrow \mathrm{A}_{\mathrm{dR}} \widehat{\otimes} \mathbb{Q}_{p}$ equals the usual embedding $\bar{K} \hookrightarrow \mathrm{B}_{\mathrm{dR}}^{+}$.

\footnotetext{
${ }^{5}$ Use the fact that every complex of abelian groups splits, i.e., is quasi-isomorphic to a complex with zero differential.
} 
(ii) For a finite extension $K^{\prime} / K, K^{\prime} \subset \bar{K}$, the evident map $A_{\mathrm{dR} \bar{K} / K} \rightarrow A_{\mathrm{dR} \bar{K} / K^{\prime}}$ yields an isomorphism $A_{\mathrm{dR} K / K} \widehat{\otimes} \mathbb{Q}_{p} \stackrel{\sim}{\rightarrow} A_{\mathrm{dR} \bar{K} / K^{\prime}} \widehat{\otimes} \mathbb{Q}_{p}$ compatible with (1.5.1).

1.6. The next result, which will not be used in the rest of the article, is a reinterpretation of Colmez's theorem $\mathrm{Col}$. It would be nice to find a simpler direct proof.

Proposition. The complexes $A_{d R} / F^{i}$ are acyclic in nonzero degrees; the maps $H^{0}\left(A_{d R} / F^{i+1}\right) \rightarrow H^{0}\left(A_{d R} / F^{i}\right)$ are injective. Set $O^{\langle i\rangle}:=H^{0}\left(A_{d R} / F^{i+1}\right)$; thus $O_{\bar{K}}=O^{\langle 0\rangle} \supset O^{\langle 1\rangle} \supset \ldots$ and $\left(A_{d R} / F^{i+1}\right) \widehat{\otimes} \mathbb{Z}_{p}$ is equal to the $p$-adic completion $\hat{O}^{\langle i\rangle}$ of $O^{\langle i\rangle}$.

Proof. By 1.4(ii), the exact cohomology sequence for $0 \rightarrow \operatorname{gr}_{F}^{i} \mathrm{~A}_{\mathrm{dR}} \rightarrow \mathrm{A}_{\mathrm{dR}} / F^{i+1}$ $\rightarrow \mathrm{A}_{\mathrm{dR}} / F^{i} \rightarrow 0$ reduces to $0 \rightarrow O^{\langle i\rangle} \rightarrow O^{\langle i-1\rangle} \stackrel{d^{\langle i\rangle}}{\rightarrow} \Omega^{\langle i\rangle} \rightarrow H^{1}\left(\mathrm{~A}_{\mathrm{dR}} / F^{i+1}\right) \rightarrow$ $H^{1}\left(\mathrm{~A}_{\mathrm{dR}} / F^{i}\right) \rightarrow 0$. So $O_{\bar{K}}=O^{\langle 0\rangle} \supset O^{\langle 1\rangle} \supset \ldots$, and the vanishing of $H^{1}\left(\mathrm{~A}_{\mathrm{dR}} / F^{i+1}\right)$ amounts to that of $H^{1}\left(\mathrm{~A}_{\mathrm{dR}} / F^{i}\right)$ combined with surjectivity of $d^{\langle i\rangle}: O^{\langle i-1\rangle} \rightarrow \Omega^{\langle i\rangle}$. It remains to prove that all $d^{\langle i\rangle}$ are surjective.

Recall that Colmez Col] considers a sequence of subalgebras $O_{\bar{K}}=O^{(0)} \supset$ $O^{(1)} \supset \ldots$ and derivations $d^{(i)}: O^{(i-1)} \rightarrow \Omega^{(i)}$ defined by induction: $d^{(i)}$ is a universal $O_{K}$-linear derivation with values in an $O_{\bar{K}}$-module, and $O^{(i)}:=\operatorname{Ker} d^{(i)}$. An induction by $i$ shows that $O^{\langle i\rangle} \supset O^{(i)}$ : Indeed, $\Omega^{\langle i\rangle}$ are $O_{\bar{K}}$-modules and $d^{\langle i\rangle}: O^{\langle i-1\rangle} \rightarrow \Omega^{\langle i\rangle}$ is a derivation; so, if $O^{\langle i-1\rangle} \supset O^{(i-1)}$, then $\left.d^{\langle i\rangle}\right|_{O^{(i-1)}}=a^{(i)} d^{(i)}$ for some $O_{\bar{K}}$-linear map $a^{(i)}: \Omega^{(i)} \rightarrow \Omega^{\langle i\rangle}$; thus $O^{\langle i\rangle} \supset O^{(i)}$.

Let $i$ be the smallest number such that $d^{\langle i\rangle}$ is not surjective. Since $E:=$ $\Omega^{\langle i\rangle} / d^{\langle i\rangle}\left(O^{\langle i-1\rangle}\right)$ is $p$-torsion $p$-divisible, one has $E \widehat{\otimes} \mathbb{Q}_{p}=T_{p} E \otimes \mathbb{Q} \neq 0$. Applying $\cdot \widehat{\otimes} \mathbb{Z}_{p}$ to the exact triangle $O^{\langle i\rangle} \rightarrow \mathrm{A}_{\mathrm{dR}} / F^{i+1} \rightarrow E[-1]$, we get a short exact sequence $0 \rightarrow \hat{O}^{\langle i\rangle} \rightarrow\left(\mathrm{A}_{\mathrm{dR}} / F^{i+1}\right) \widehat{\otimes} \mathbb{Z}_{p} \rightarrow T_{p} E \rightarrow 0$. By [Col], $\mathrm{A}_{\text {inf }} / F^{i+1}=\hat{O}^{(i)}$. By universality, the map $u_{i}: \mathrm{A}_{\text {inf }} / F^{i+1} \rightarrow\left(\mathrm{A}_{\mathrm{dR}} / F^{i+1}\right) \widehat{\otimes} \mathbb{Z}_{p}$ equals the composition $\hat{O}^{(i)} \rightarrow \hat{O}^{\langle i\rangle} \hookrightarrow\left(\mathrm{A}_{\mathrm{dR}} / F^{i+1}\right) \widehat{\otimes} \mathbb{Z}_{p}$, so its composition with the projection onto $T_{p} E$ vanishes. This cannot happen since $u_{i \mathbb{Q}}$ is an isomorphism (see $\S 1.5$ ), q.e.d.

\section{2. h-TOPOLOGY AND SEMI-STABLE COMPACTIFICATIONS}

2.1. A topological digression. The next proposition is a generalization of [V2, 4.1].

Let $\mathcal{V}$ be an essentially small site. As in [V1], we denote by $\mathcal{V}^{\sim}$ the corresponding topos (the category of sheaves of sets on $\mathcal{V}$ ).

For us, a base for $\mathcal{V}$ is a pair $(\mathcal{B}, \phi)$, where $\mathcal{B}$ is an essentially small category and $\phi: \mathcal{B} \rightarrow \mathcal{V}$ is a faithful functor, that satisfies the next property:

$(*)$ For any $V \in \mathcal{V}$ and a finite family of pairs $\left(B_{\alpha}, f_{\alpha}\right), B_{\alpha} \in \mathcal{B}, f_{\alpha}: V \rightarrow$ $\phi\left(B_{\alpha}\right)$, there exists a set of objects $B_{\beta}^{\prime} \in \mathcal{B}$ and a covering family $\left\{\phi\left(B_{\beta}^{\prime}\right) \rightarrow\right.$ $V\}$ such that every composition $\phi\left(B_{\beta}^{\prime}\right) \rightarrow V \rightarrow \phi\left(B_{\alpha}\right)$ lies in $\operatorname{Hom}\left(B_{\beta}^{\prime}, B_{\alpha}\right) \subset$ $\operatorname{Hom}\left(\phi\left(B_{\beta}^{\prime}\right), \phi\left(B_{\alpha}\right)\right)$.

Remarks. (i) Property $(*)$ for an empty set of $\left(B_{\alpha}, f_{\alpha}\right)$ 's means that every $V \in \mathcal{V}$ has a covering by objects $\phi(B), B \in \mathcal{B}$. If $\phi$ is fully faithful, then $(*)$ amounts to this assertion 6

(ii) If $\mathcal{B}$ admits finite products and $\phi$ commutes with finite products, then it suffices to check $(*)$ for families $\left(B_{\alpha}, f_{\alpha}\right)$ having $\leq 1$ element.

${ }^{6}$ The proposition below in this situation amounts to [V2, 4.1]. 
(iii) In the general case, it suffices to check $(*)$ for families $\left(B_{\alpha}, f_{\alpha}\right)$ having $\leq 2$ elements.

Suppose $(\mathcal{B}, \phi)$ is a base for $\mathcal{V}$. Define a covering sieve in $\mathcal{B}$ as a sieve whose $\phi$-image is a covering family in $\mathcal{V}$.

Proposition. (i) Covering sieves in $\mathcal{B}$ form a Grothendieck topology on $\mathcal{B}$.

(ii) The functor $\phi: \mathcal{B} \rightarrow \mathcal{V}$ is continuous (see $[\mathrm{V} 2,1.1]$ ).

(iii) $\phi$ yields an equivalence of the toposes: one has $\mathcal{B}^{\sim} \stackrel{\sim}{\rightarrow} \mathcal{V}^{\sim}$.

We call the above topology on $\mathcal{B}$ the $\phi$-induced topology 7

Proof. (i) Let us check that covering sieves in $\mathcal{B}$ are stable with respect to pullback; the rest of the axioms from [V1, 1.1] are evident. For a morphism $g: B^{\prime} \rightarrow B$ in $\mathcal{B}$ and a covering sieve $\mathfrak{s}$ on $B$, let us find a covering family on $B^{\prime}$ that belongs to the $g$ pullback of $\mathfrak{s}$. The $\phi(g)$-pullback of $\phi(\mathfrak{s})$ is a covering sieve in $\mathcal{V}$, so there is a covering family $\left\{\pi_{\gamma}: V_{\gamma} \rightarrow \phi\left(B^{\prime}\right)\right\}$ such that every composition $V_{\gamma} \rightarrow \phi\left(B^{\prime}\right) \rightarrow \phi(B)$ can be factored as $V_{\gamma} \stackrel{g_{\gamma}}{\longrightarrow} \phi\left(B_{\gamma}\right) \stackrel{\phi\left(p_{\gamma}\right)}{\longrightarrow} \phi(B)$, where $p_{\gamma}: B_{\gamma} \rightarrow B$ belong to $\mathfrak{s}$. Applying $(*)$ to $V_{\gamma}$ and $\left(B^{\prime}, \pi_{\gamma}\right),\left(B_{\gamma}, g_{\gamma}\right)$, we find a covering family $\left\{\phi\left(B_{\beta_{\gamma}}^{\prime}\right) \rightarrow V_{\gamma}\right\}$ as in $(*)$. The composite covering $\left\{\phi\left(B_{\beta_{\gamma}}^{\prime}\right) \rightarrow \phi\left(B^{\prime}\right)\right\}$ comes then from a covering family $\left\{B_{\beta_{\gamma}}^{\prime} \rightarrow B^{\prime}\right\}$ in $\mathcal{B}$ which lies in the $g$-pullback of $\mathfrak{s}$.

(ii) We know that $\phi$ sends covering families to covering families, so it suffices to show that for any given $p_{\alpha}: B_{\alpha} \rightarrow B$ in $\mathcal{B}$ and $f_{\alpha}: V \rightarrow \phi\left(B_{\alpha}\right), \alpha=1,2$, such that $\phi\left(p_{1}\right) f_{1}=\phi\left(p_{2}\right) f_{2}$ there is a covering $\left\{\pi_{\beta}: V_{\beta} \rightarrow V\right\}$ and morphisms $\xi_{\alpha \beta}: B_{\beta}^{\prime} \rightarrow$ $B_{\alpha}, g_{\beta}: V_{\beta} \rightarrow \phi\left(B_{\beta}^{\prime}\right)$ such that $p_{1} \xi_{1 \beta}=p_{2} \xi_{2 \beta}$ and $\phi\left(\xi_{\alpha \beta}\right) g_{\beta}=f_{\alpha} \pi_{\beta}$. Such a datum (with $g_{\beta}$ the identity map) comes from $(*)$ applied to $V$ and $\left(B_{1}, f_{1}\right),\left(B_{2}, f_{2}\right)$.

(iii) By (ii), one has the usual adjoint functors between the categories of sheaves $\left(\phi^{s}, \phi_{s}\right): \mathcal{B}^{\sim} \leftrightarrows \mathcal{V}^{\sim}$. To prove that they are mutually inverse equivalences, we will check that for $\mathcal{F} \in \mathcal{B}^{\sim}$ and $\mathcal{G} \in \mathcal{V}^{\sim}$ the adjunction maps $a_{\mathcal{F}}: \mathcal{F} \rightarrow \phi_{s} \phi^{s} \mathcal{F}$, $b_{\mathcal{G}}: \phi^{s} \phi_{s} \mathcal{G} \rightarrow \mathcal{G}$ are isomorphisms.

Recall that $\phi^{s} \mathcal{F}=(\phi \mathcal{F})^{\sim}$, where $\phi$ is the pullback of presheaves and $\sim$ is the sheafification functor. For $V \in \mathcal{V}$ one has $(\phi \mathcal{F})(V)=\operatorname{colim}_{\mathcal{C}(V)} \mathcal{F}$, where $\mathcal{C}(V)$ is the category of pairs $(B, f), B \in \mathcal{B}, f: V \rightarrow \phi(B)$, with $\operatorname{Hom}_{\mathcal{C}(V)}\left((B, f),\left(B^{\prime}, f^{\prime}\right)\right)$ $:=\left\{g \in \operatorname{Hom}\left(B^{\prime}, B\right): \phi(g) f^{\prime}=f\right\}$, and we set $\mathcal{F}(B, f):=\mathcal{F}(B)$.

(a) To show that $a_{\mathcal{F}}$ is an isomorphism, we check that it is injective and surjective:

$a_{\mathcal{F}}$ is injective: Suppose we have $B \in \mathcal{B}$ and $\xi_{1}, \xi_{2} \in \mathcal{F}(B)$ such that $a_{\mathcal{F}}\left(\xi_{1}\right)=$ $a_{\mathcal{F}}\left(\xi_{2}\right)$; let us show that the $\xi_{i}$ coincide. One has

$$
a_{\mathcal{F}}\left(\xi_{i}\right) \in\left(\phi_{s} \phi^{s} \mathcal{F}\right)(B)=\left(\phi^{s} \mathcal{F}\right)(\phi(B)),
$$

and the equality means that there is a covering $\left\{\pi_{\gamma}: V_{\gamma} \rightarrow \phi(B)\right\}$ such that the images of $\xi_{i}$ in $(\phi \mathcal{F})\left(V_{\gamma}\right)=\operatorname{colim}_{\mathcal{C}\left(V_{\gamma}\right)} \mathcal{F}$ coincide. Thus for some finite subdiagram $\mathcal{C}\left(V_{\gamma}\right)^{\prime} \subset \mathcal{C}\left(V_{\gamma}\right)$ that contains $\left(B, \pi_{\gamma}\right)$ the images of $\xi_{i}$ in $\operatorname{colim}_{\mathcal{C}\left(V_{\gamma}\right)^{\prime}} \mathcal{F}$ coincide. Applying $(*)$ to $V_{\gamma}$ and pairs from $\mathcal{C}\left(V_{\gamma}\right)^{\prime}$, we get a covering $\left\{\phi\left(B_{\beta_{\gamma}}^{\prime}\right) \rightarrow V_{\gamma}\right\}$ such that the image of $\mathcal{C}\left(V_{\gamma}\right)^{\prime}$ in each $\mathcal{C}\left(\phi\left(B_{\beta_{\gamma}}^{\prime}\right)\right)$ comes from a diagram in $\mathcal{B}^{\circ} / B_{\beta_{\gamma}}^{\prime}$. The composite covering $\left\{\phi\left(B_{\beta_{\gamma}}^{\prime}\right) \rightarrow \phi(B)\right\}$ comes then from a covering $\left\{B_{\beta_{\gamma}}^{\prime} \rightarrow B\right\}$ in $\mathcal{B}$, and the images of $\xi_{i}$ in $\mathcal{F}\left(B_{\beta_{\gamma}}^{\prime}\right)$ coincide. Then $\xi_{1}=\xi_{2}$ since $\mathcal{F}$ is a sheaf, q.e.d.

\footnotetext{
${ }^{7}$ The terminology is compatible with that of [V2 3.1].
} 
$a_{\mathcal{F}}$ is surjective: For $B \in \mathcal{B}, \chi \in\left(\phi_{s} \phi^{s} \mathcal{F}\right)(B)$ we look for a covering $\left\{B_{\beta}^{\prime} \rightarrow B\right\}$ in $\mathcal{B}$ such that $\left.\chi\right|_{B_{\beta}^{\prime}}$ lies in the image of $\mathcal{F}\left(B_{\beta}^{\prime}\right) \rightarrow\left(\phi_{s} \phi^{s} \mathcal{F}\right)\left(B_{\beta}^{\prime}\right)$. To find it, consider $\chi$ as an element of $\left(\phi^{s} \mathcal{F}\right)(\phi(B))$. There is a covering $\left\{\pi_{\gamma}: V_{\gamma} \rightarrow \phi(B)\right\}$ such that $\left.\chi\right|_{V_{\gamma}}$ lies in the image of $(\phi \mathcal{F})\left(V_{\gamma}\right) \rightarrow\left(\phi^{s} \mathcal{F}\right)\left(V_{\gamma}\right)$; i.e., one has $f_{\gamma}: V_{\gamma} \rightarrow \phi\left(B_{\gamma}\right)$ such that $\left.\chi\right|_{V_{\gamma}}$ lies in the image of the composition $\mathcal{F}\left(B_{\gamma}\right) \rightarrow\left(\phi^{s} \mathcal{F}\right)\left(\phi\left(B_{\gamma}\right)\right) \rightarrow\left(\phi^{s} \mathcal{F}\right)\left(V_{\gamma}\right)$, where the second arrow comes from $f_{\gamma}$. Applying $(*)$ to $V_{\gamma}$ and $\left(B, \pi_{\gamma}\right),\left(B_{\gamma}, f_{\gamma}\right)$, we find a covering $\left\{\phi\left(B_{\beta_{\gamma}}^{\prime}\right) \rightarrow V_{\gamma}\right\}$ as in $(*)$; the composite covering $\left\{\phi\left(B_{\beta_{\gamma}}^{\prime}\right) \rightarrow \phi(B)\right\}$ comes then from a covering $\left\{B_{\beta_{\gamma}}^{\prime} \rightarrow B\right\}$ that satisfies the promised property.

(b) $b_{\mathcal{G}}$ is an isomorphism: Since $\phi_{s}\left(b_{\mathcal{G}}\right) a_{\phi_{s} \mathcal{G}}=\mathrm{id}_{\phi_{s} \mathcal{G}}$ and we already know that $a_{\phi_{s} \mathcal{G}}$ is an isomorphism, we see that $\phi_{s}\left(b_{\mathcal{G}}\right): \phi_{s} \phi^{s} \phi_{s}(\mathcal{G}) \rightarrow \phi_{s} \mathcal{G}$ is an isomorphism. Thus $b_{\mathcal{G}}(B): \phi^{s} \phi_{s} \mathcal{G}(\phi(B)) \rightarrow \mathcal{G}(\phi(B))$ is an isomorphism for every $B \in \mathcal{B}$. Since every $V \in \mathcal{V}$ admits a covering by objects $\phi(B), B \in \mathcal{B}$, this implies that $b_{\mathcal{G}}$ is both injective and surjective, hence an isomorphism, q.e.d.

Exercises. (i) For any presheaf $\mathcal{J}$ on $\mathcal{V}$ one has $\phi_{s}\left(\mathcal{J}^{\sim}\right)=(\phi . \mathcal{J})^{\sim}$.

(ii) Suppose $(\mathcal{B}, \phi)$ is a base for $\mathcal{V}$ and $\left(\mathcal{B}^{\prime}, \phi^{\prime}\right)$ is a base for the $\phi$-induced topology on $\mathcal{B}$. Then $\left(\mathcal{B}^{\prime}, \phi \phi^{\prime}\right)$ is a base for $\mathcal{V}$.

2.2. For a field $K$, let $\mathcal{V} a r_{K}$ be the category of $K$-varieties, i.e., reduced separated $K$-schemes of finite type. We will consider categories $\mathcal{B}$ formed by varieties equipped with appropriate compactifications, referred to as pairs:

(a) Geometric setting: Let $j: U \hookrightarrow \bar{U}$ be an open embedding such that $\bar{U}$ is proper and $U$ is dense in $\bar{U}$. We call such a datum a geometric pair over $K$, or geometric $K$-pair, and denote it by $(U, \bar{U})$. We say that $(U, \bar{U})$ is a regular normal crossings pair, nc-pair for short, if $\bar{U}$ is a regular scheme and $\bar{U} \backslash U$ is a divisor with normal crossings in $\bar{U}$; it is a strict nc-pair if the irreducible components of $\bar{U} \backslash U$ are regular. A morphism $f:\left(U^{\prime}, \bar{U}^{\prime}\right) \rightarrow(U, \bar{U})$ of pairs is a map $\bar{U}^{\prime} \rightarrow \bar{U}$ which sends $U^{\prime}$ to $U$. We denote the category of geometric $K$-pairs by $\mathcal{V} a r_{K}^{c}$; let $\mathcal{V} a r_{K}^{\mathrm{nc}}$ be the full subcategory of nc-pairs.

(b) Arithmetic $K$-setting: Suppose $K$ is a $p$-adic field as in $\S 1.3$. An arithmetic pair over $K$, a.k.a. arithmetic $K$-pair, is an open embedding $j: U \hookrightarrow \bar{U}$ with dense image of a $K$-variety $U$ into a reduced proper flat $O_{K}$-scheme $\bar{U}$.

For such a $(U, \bar{U})$ we set $O_{K_{U}}:=\Gamma\left(\bar{U}, \mathcal{O}_{\bar{U}}\right), K_{U}:=\Gamma\left(\bar{U}_{K}, \mathcal{O}_{\bar{U}}\right)$. Then $K_{U}$ is the product of several finite extensions of $K$ (labeled by the connected components of $\bar{U}_{K}$; if $\bar{U}$ is normal, then $O_{K_{U}}$ is the product of the corresponding rings of integers. The closed fiber $\bar{U}_{s}$ of $\bar{U}$ is the union of fibers over the closed points of $O_{K_{U}}$.

We say that $(U, \bar{U})$ is a semi-stable pair, or simply ss-pair, if (i) $\bar{U}$ is a regular scheme, (ii) $\bar{U} \backslash U$ is a divisor with normal crossings on $\bar{U}$, and (iii) the closed fiber $\bar{U}_{s}$ is reduced. Our ss-pair is strict if the irreducible components of $\bar{U} \backslash U$ are regular. Arithmetic $K$-pairs form a category $\mathcal{V} a r_{K}^{c c}$; let $\mathcal{V} a r_{K}^{\mathrm{ss}}$ be the full subcategory of ss-pairs.

(c) Arithmetic $\bar{K}$-setting: For $K$ as in (b), let $\bar{K}$ be its algebraic closure. An arithmetic pair over $\bar{K}$, a.k.a. arithmetic $\bar{K}$-pair, is an open embedding $j: V \hookrightarrow \bar{V}$ with dense image of a $\bar{K}$-variety $V$ into a reduced proper flat $O_{\bar{K}}$-scheme $\bar{V}$. A connected $(V, \bar{V})$ is said to be semi-stable, a.k.a. ss-pair, if there exists an ss-pair $(U, \bar{U})$ over $K$ and a $\bar{K}$-point $\alpha: K_{U} \rightarrow \bar{K}$ (see (b)) such that $(V, \bar{V})$ is isomorphic to $(U, \bar{U})_{\alpha}=\left(U_{\bar{K}}, \bar{U}_{O_{\bar{K}}}\right):=\left(U \otimes_{K_{U}} \bar{K}, \bar{U} \otimes_{O_{K_{U}}} O_{\bar{K}}\right)$. Then $\bar{V}$ is normal (say, by Serre's criterion). An arbitrary $(V, \bar{V})$ is semi-stable if such are all its connected 
components. Denote by $\mathcal{V} a r_{\bar{K}}^{c c}$ the category of all arithmetic pairs over $\bar{K}$, and by $\mathcal{V} a r_{\bar{K}}^{\mathrm{ss}}=\mathcal{V} a r_{\bar{K} / K}^{\mathrm{ss}}$ its full subcategory of ss-pairs.

Remark. If $K^{\prime}$ is a finite extension of $K$ contained in $\bar{K}$, then $\mathcal{V} a r_{\bar{K} / K^{\prime}}^{\mathrm{ss}} \subset \mathcal{V} a r_{\bar{K} / K}^{\mathrm{ss}}$. For all the constructions below the difference between them is irrelevant.

These categories are connected by commutative diagrams of functors

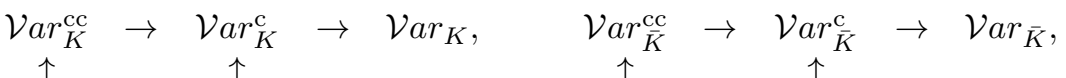

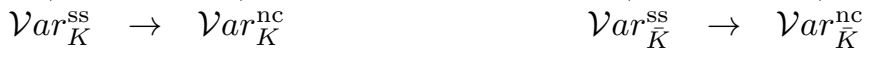

where the vertical arrows are the fully faithful embeddings, and the upper horizontal lines are faithful forgetful functors of passing to the generic fiber and $(U, \bar{U}) \mapsto U$. The $K$ - and $\bar{K}$-settings are connected by base change functors

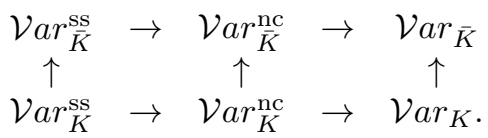

Here the two right vertical arrows are the evident base change $\cdot \otimes_{K} \bar{K}$, and the left one assigns to a semi-stable $K$-pair $(U, \bar{U})$ the disjoint sum of pairs $(U, \bar{U})_{\alpha}$ for all $\bar{K}$-points $\alpha: K_{U} \rightarrow \bar{K}$.

2.3. A morphism $f:(V, \bar{V}) \rightarrow(U, \bar{U})$ of pairs in either of the settings of $\S 2.2$ is called an alteration (of $(U, \bar{U})$ ) if $f^{-1}(U)=V$, the generic fibers of $f$ are zero-dimensional, and their union is dense in $V$. In setting (a), $f$ is a (strict) nc-alteration if $(V, \bar{V})$ is a (strict) nc-pair; in settings (b), (c), $f$ is a (strict) ss-alteration if $(V, \bar{V})$ is a (strict) ss-pair.

If $f$ is an alteration, then $\left.f\right|_{V}: V \rightarrow U$ is proper and surjective; the composition of alterations is an alteration.

Here is a key result of de Jong [J1, 4.1, 6.5]:

Theorem. Every geometric pair admits a strict nc-alteration. Every arithmetic pair, either over $K$ or over $\bar{K}$, admits a strict ss-alteration. The alterations can be chosen so that $\bar{V}$ is projective.

Remark. Our conventions slightly differ from de Jong's: he understands varieties to be irreducible and semi-stable $K$-pairs $(U, \bar{U})$ to have property $K_{U}=K$; his notation for $(U, \bar{U})$ is $(\bar{U}, Z), Z:=\bar{U} \backslash U$.

2.4. For a field $K$, the $h$-topology (see [SV]) on $\mathcal{V} r_{K}$ is generated by the pretopology whose coverings are finite families of maps $\left\{Y_{\alpha} \rightarrow X\right\}$ such that $Y:=\amalg Y_{\alpha} \rightarrow X$ is a universal topological epimorphism 8 It is stronger than the étale and proper topologies 9 We denote the h-site by $\mathcal{V} a r_{K \mathrm{~h}}$; the h-site of $X$ is denoted by $X_{\mathrm{h}}$.

Exercise. Let $f: Y \rightarrow X$ be a morphism in $\mathcal{V} r_{K}$.

(i) $f$ is an h-covering if (and only if) for every irreducible curve $C \subset X$ the base change $Y_{\tilde{C}} \rightarrow \tilde{C}$, where $\tilde{C}$ is the normalization of $C$, is an h-covering 10

\footnotetext{
${ }^{8}$ This means that a subset of $X$ is Zariski open if (and only if) its preimage in $Y$ is open, and the same is true after any base change.

${ }^{9}$ The latter is generated by a pretopology whose coverings are proper surjective maps.

${ }^{10}$ Hint: For an open $V \subset Y$ its image in $X$ is constructible (EGA IV 1.8.4), so to show that $f(V)$ is open it suffices to check that for any curve $C \subset X$ the intersection $C \cap f(V)$ is open in $C$.
} 
(ii) If $X$ is a regular curve, then $f$ is an h-covering if (and only if) the closure of the generic fiber of $f$ maps onto $X$, or, equivalently, $f$ is a covering for the flat topology.

Remark. By SV 10.4], every h-covering is a Zariski covering locally in proper topology. Therefore (see $[\mathrm{D}],[\mathrm{SD}$, or $[\mathrm{Con}]$ ) h-coverings are morphisms of universal cohomological descent for torsion étale sheaves; if $K=\mathbb{C}$, then h-coverings are morphisms of universal cohomological descent for arbitrary sheaves on the classical topology. In particular, for any h-hypercovering $Y$. of $X$ and an abelian group $A$ the canonical map $R \Gamma\left(X_{\text {ét }}, A\right) \rightarrow R \Gamma\left(Y_{\text {ét }}, A\right)(:=$ the total complex of the cosimplicial system of complexes $\left.R \Gamma\left(Y_{i \text { ét }}, A\right)\right)$ is a quasi-isomorphism if $A$ is a torsion group. Passing to the limit, we see that $R \Gamma_{\text {ét }}\left(X, \mathbb{Z}_{p}\right) \stackrel{\sim}{\rightarrow} R \Gamma_{\text {ét }}\left(Y\right.$., $\left.\mathbb{Z}_{p}\right)$. If $K=\mathbb{C}$, then $R \Gamma\left(X_{\mathrm{cl}}, A\right) \stackrel{\sim}{\rightarrow} R \Gamma\left(Y_{\text {.cl }}, A\right)$ for any $A$. Since h-topology is stronger than the étale one, we see that $R \Gamma\left(X_{\text {ét }}, A\right) \stackrel{\sim}{\rightarrow} R \Gamma\left(X_{\mathrm{h}}, A\right)$ if $A$ is a torsion group (see [SV], 10.7] for a direct proof).

2.5. Let $\phi$ be the forgetful functor $(U, \bar{U}) \mapsto U$ on any of the categories $\mathcal{V} a r^{\text {? }}$ in $\S 2.2$.

Proposition. If $\mathcal{V} r^{?}$ is either of the categories $\mathcal{V}_{K}^{c}, \mathcal{V} a r_{K}^{n c}, \mathcal{V} a r_{K}^{c c}, \mathcal{V}_{K}^{\mathrm{ss}}$, then

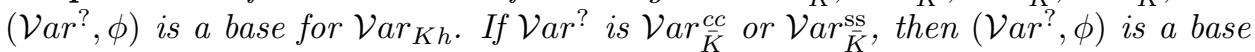
for $\operatorname{Var}_{\bar{K} h}$.

Proof. We consider the arithmetic $K$-setting, leaving the other two settings for the reader. Let us show that $\left(\mathcal{V}_{K}^{\mathrm{cc}}, \phi\right)$ satisfies condition $(*)$ from $\S 2.1$. Our datum is a $K$-variety $V$ and a finite collection of arithmetic $K$-pairs $\left(U_{\alpha}, \bar{U}_{\alpha}\right)$ and maps $f_{\alpha}: V \rightarrow U_{\alpha}$. We need to find an h-covering $\pi: V^{\prime} \rightarrow V$ and an arithmetic pair $\left(V^{\prime}, \bar{V}^{\prime}\right)$ such that $f_{\alpha} \pi$ extend to maps $\left(V^{\prime}, \bar{V}^{\prime}\right) \rightarrow\left(U_{\alpha}, \bar{U}_{\alpha}\right)$. First we find an hcovering $V^{\prime} \rightarrow V$ such that $V^{\prime}$ sits in some arithmetic $K$-pair $\left(V^{\prime}, \tilde{V}^{\prime}\right)$ : let $V^{\prime} / V$ be a quasi-projective modification of $V$ provided by the Chow lemma, and take for $\tilde{V}^{\prime}$ the closure of $V^{\prime}$ in a projective space 11 Then take $\bar{V}^{\prime}$ to be the closure of the image of $V^{\prime}$ by the embedding $V^{\prime} \hookrightarrow \tilde{V}^{\prime} \times \Pi \bar{U}_{\alpha}$, and we are done.

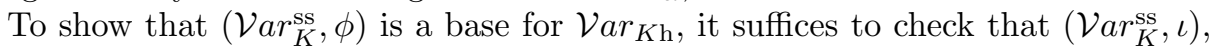
where $\iota$ is the embedding $\mathcal{V} a r_{K}^{\mathrm{ss}} \hookrightarrow \mathcal{V} a r_{K}^{\mathrm{cc}}$, is a base for the $\phi$-induced topology on $\mathcal{V} r_{K}^{c c}$ (see Exercise (ii) in $\S 2.1$ ). Since $\iota$ is fully faithful, it suffices to check that for every $(U, \bar{U}) \in \mathcal{V} a r_{K}^{c c}$ there exists a map $\left(U^{\prime}, \bar{U}^{\prime}\right) \rightarrow(U, \bar{U})$ such that $U^{\prime} \rightarrow U$ is an h-covering and $\left(U^{\prime}, \bar{U}^{\prime}\right)$ is semi-stable. Such a datum is provided by the de Jong theorem in $\S 2.3$, and we are done.

We call the $\phi$-induced topology on either of the categories $\mathcal{V} a r$ ? the $h$-topology.

Remarks. (i) Any h-covering of $(U, \bar{U}) \in \mathcal{V} a r_{K}^{\text {ss }}$ has a refinement with terms of the same dimension as $U$ (indeed, the same assertion in $\mathcal{V} a r_{K}$ is true by [SV, 10.4]; to pass to $\mathcal{V} a r_{K}^{\text {ss }}$, we apply the constructions from the proof above, and they preserve the dimension).

(ii) The proposition remains true if we replace the category of ss- or nc-pairs by its subcategory of strict pairs $(U, \bar{U})$ with projective $\bar{U}$.

(iii) For any functor in (2.2.1) its source is a base for the h-topology of the target, and the induced topology on the source is the h-topology.

(iv) The functors in (2.2.2) are continuous for the h-topologies.

\footnotetext{
${ }^{11}$ In fact, every $V$ sits in a $K$-pair due to Nagata's theorem.
} 
2.6. By $\S 2.1$ and $\S 2.5, \phi$ identifies h-sheaves on $\mathcal{V} a r_{K}$, resp. $\mathcal{V} a r_{\bar{K}}$, with h-sheaves on $\mathcal{V} a r_{K}^{\mathrm{c}}, \mathcal{V} a r_{K}^{\mathrm{nc}}, \mathcal{V} a r_{K}^{\mathrm{cc}}, \mathcal{V} a r_{K}^{\mathrm{ss}}$, resp. $\mathcal{V}_{\bar{K}}^{\mathrm{cc}}, \mathcal{V} a r_{\bar{K}}^{\mathrm{ss}}$. Thus we have the $h$-localization functors

$$
\mathcal{P S h}\left({\mathcal{V} a r_{K}^{?}}^{?}\right) \rightarrow \mathcal{V} a r_{\overline{K h}}, \quad \mathcal{P} \mathcal{S h}\left(\mathcal{V}_{a} r_{\bar{K}}^{?}\right) \rightarrow \mathcal{V} a r_{\bar{K} \mathrm{~h}}
$$

which assign to any presheaf $\mathcal{F}$ on pairs the corresponding h-sheaf $\mathcal{F}^{\sim}$ viewed as an h-sheaf on varieties.

Remark. For any presheaf on $\mathcal{V} a r_{K}^{\mathrm{c}}, \mathcal{V} a r_{K}^{\mathrm{cc}}$ or $\mathcal{V} a r_{\bar{K}}^{\mathrm{cc}}$, its h-sheafification coincides with h-sheafification of its restriction to resp. $\mathcal{V}_{K}^{\mathrm{nc}}, \mathcal{V} a r_{K}^{\mathrm{ss}}$ or $\mathcal{V} a r_{\bar{K}}^{\mathrm{ss}}$. For a presheaf on $\mathcal{V}_{a} r_{\bar{K} / K}^{\mathrm{ss}}$, its h-sheafification is the same as h-sheafification of its restriction to $\mathcal{V} a r_{\bar{K} / K^{\prime}}^{\mathrm{ss}}$, where $K^{\prime} \subset \bar{K}$ is any finite extension of $K$ (see Remark in $\S 2.2$ ).

\section{THE $p$-ADIC PERIOD MAP}

3.1. The derived de Rham algebra in logarithmic setting. We refer to [K1 for log scheme basics. There are two (in general, nonequivalent) ways to define the cotangent complex for log schemes due, respectively, to Gabber and Olsson; see O1] 12 Gabber's approach $([\mathrm{Ol}, \S 8])$ is more direct and precise 13 we recall it briefly.

For a commutative ring $A$, a prelog structure on $A$ is a homomorphism of monoids $\alpha: L \rightarrow A$, where $L$ is a commutative integral monoid (written multiplicatively) and $A$ is viewed as a monoid with respect to the product. Rings equipped with prelog structures form a category in an evident way; denote its objects simply by $(A, L)$. For a fixed $(A, L)$, let $\mathcal{C}_{(A, L)}$ be the category of morphisms $(A, L) \rightarrow(B, M)$; we denote such an object by $(B, M) /(A, L)$. Let $\Omega_{(B, M) /(A, L)}$ be the $B$-module of relative Kähler log differentials: it is generated by $\Omega_{B / A}$ and elements $d \log m, m \in$ $M$, subject to relations $d \log \left(m_{1} m_{2}\right)=d \log m_{1}+d \log m_{2}, \alpha(m) d \log m=d \alpha(m)$, and $d \log m=0$ if $m$ is in the image of $L$. The de Rham dg algebra of relative $\log$ forms $\Omega_{(B, M) /(A, L)}$ has components $\Omega_{(B, M) /(A, L)}^{i}:=\Lambda_{B}^{i} \Omega_{(B, M) /(A, L)}$; elements $d \log m$ are degree 1 cycles. It carries the Hodge filtration $F^{n}=\Omega_{(B, M) /(A, L)}^{\geq n}$.

A pair of sets $I, J$ yields a free object $P_{(A, L)}[I, J]$ in $\mathcal{C}_{(A, L)}$ : the corresponding ring is a polynomial algebra $A\left[t_{i}, t_{j}\right]_{i \in I, j \in J}$, the monoid is $L \oplus \mathbb{N}[I]$, where $\mathbb{N}[I]$ is the free monoid generated by $I$, and the structure map sends the generator $m_{i}$ of $\mathbb{N}[I]$, $i \in I$, to $t_{i}$. The de Rham algebra $\Omega_{P_{(A, L)}[I, J] /(A, L)}$ is freely generated, as a graded commutative $A$-algebra, by elements $t_{i}, t_{j}$ of degree 0 and $d \log t_{i}:=d \log m_{i}, d t_{j}$ of degree 1 , where $i \in I, j \in J$.

Every $(B, M) /(A, L) \in \mathcal{C}_{(A, L)}$ admits a canonical simplicial resolution $P$. $=$ $P_{(A, L)}(B, M)$.. This is a simplicial object of $\mathcal{C}_{(A, L)}$ augmented over the object $(B, M) /(A, L)$ and such that every $P_{i}$ is a free object as above. Thus we have the simplicial dg algebra $\Omega_{P . /(A, L)}$ filtered by the Hodge filtration $F$. Denote by $L \Omega_{(B, M) /(A, L)}$ the corresponding total complex, $L \Omega_{(B, M) /(A, L)}^{a}=\bigoplus_{j-i=a} \Omega_{P_{i} /(A, L)}^{j}$; this is a filtered commutative dg algebra. Let $L \Omega_{(\hat{B}, M) /(A, L)}$ be its $F$-completion; as in $\S 1.2$, we understand it as a mere projective system of quotients $L \Omega_{(\hat{B}, M) /(A, L)} / F^{n}$. One has a natural quasi-isomorphism of graded dg algebras $\operatorname{gr}_{F}^{*} L \Omega_{(\hat{B}, M) /(A, L)} \stackrel{\sim}{\rightarrow}$ $\left(L \Lambda_{B}^{*}\left(\mathrm{~L}_{(B, M) /(A, L)}\right)\right)[-*]$. Here $\mathrm{L}_{(B, M) /(A, L)}:=\Omega_{P . /(A, L)} \otimes_{P} . B$ is the relative

\footnotetext{
${ }^{12}$ In all situations that we will consider, the two versions coincide by [Ol, 8.34].

${ }^{13}$ It produces a true complex, while Olsson's construction yields a mere compatible datum of the canonical filtration truncations.
} 
$\log$ cotangent complex; it is acyclic in positive degrees, and $H^{0} \mathrm{~L}_{(B, M) /(A, L)}=$ $\Omega_{(B, M) /(A, L)}$. The constructions are compatible with direct limits. If in the above definition we replace $P$. by any free simplicial resolution of $(B, M) /(A, L)$, then the output is naturally quasi-isomorphic to $L \Omega_{\left({ }_{B}, M\right) /(A, L)}$. The plain cotangent complex and derived de Rham algebra for $B / A$ map naturally to logarithmic ones.

For any map $(X, \mathcal{M}) \rightarrow(S, \mathcal{L})$ of integral $\log$ schemes, the above construction, being étale sheafified, yields the log cotangent complex $\mathrm{L}_{(X, \mathcal{M}) /(S, \mathcal{L})}$, the derived $\log$ de Rham algebra $L \Omega_{(X, \mathcal{M}) /(S, \mathcal{L})}$, and its $F$-completion $L \Omega^{\circ}(\hat{X}, \mathcal{M}) /(S, \mathcal{L})$, which are complexes of sheaves on $X_{\text {ét }}$. We use only the completed complex $L \Omega^{\wedge}$.

3.2. Let $(U, \bar{U})$ be a pair as in $\S 2.2$. We view $\bar{U}$ as a log scheme with the usual integral $\log$ structure $\mathcal{O}_{\bar{U}} \cap j_{*} \mathcal{O}_{U}^{\times} \rightarrow \mathcal{O}_{\bar{U}}$; by abuse of notation, let us denote this log scheme again by $(U, \bar{U})$. Any morphism of pairs $(U, \bar{U}) \rightarrow(V, \bar{V})$ is a morphism of $\log$ schemes, so we have the relative log cotangent complex $\mathrm{L}_{(U, \bar{U}) /(V, \bar{V})}$, the derived log de Rham algebra $L \Omega_{(\hat{U}, \bar{U}) /(V, \bar{V})}$, etc., as above. There is a canonical morphism $L \Omega^{\prime} \mathcal{U}_{/ \bar{V}} \rightarrow L \Omega_{(\hat{U}, \bar{U}) /(V, \bar{V})}$. We also have "absolute" complexes: in the arithmetic $K$ - or $\bar{K}$-setting, these are $\mathrm{L}_{(U, \bar{U})}:=\mathrm{L}_{(U, \bar{U}) / O_{K}}, L \Omega_{(\hat{U}, \bar{U})}^{\prime}:=L \Omega_{(\hat{U}, \bar{U}) / O_{K}}$, where $O_{K}$ is considered with the trivial $\log$ structure $O_{K}^{\times}$; for the geometric $K$ - or $\bar{K}$-setting, replace $O_{K}$ by $K$, resp. $\bar{K}$.

Remark. For $(V, \bar{V}) \in \mathcal{V} a r_{\bar{K}}^{\text {nc }}$ one has $L \Omega_{(\hat{V}, \bar{V})} \stackrel{\sim}{\rightarrow} \Omega_{(V, \bar{V})}$. Hence for $(U, \bar{U}) \in \mathcal{V} a r_{\bar{K}}^{\mathrm{ss}}$ one has $R \Gamma\left(\bar{U}, L \Omega_{(\hat{U}, \bar{U})}\right) \otimes \mathbb{Q} \stackrel{\sim}{\rightarrow} R \Gamma\left(\bar{U}_{\bar{K}}, \Omega_{\left(U, \bar{U}_{\bar{K}}\right)}\right)$. Ditto for pairs over $K$.

Consider now the arithmetic $\bar{K}$-pair $\operatorname{Spec}\left(\bar{K}, O_{\bar{K}}\right):=\left(\operatorname{Spec} \bar{K}, \operatorname{Spec} O_{\bar{K}}\right)$ :

Lemma. The cotangent complex $L_{\left(\bar{K}, O_{\bar{K}}\right)}$ is acyclic in nonzero degrees, and the canonical map $\Omega_{O_{\bar{K}}} \rightarrow \Omega_{\left(\bar{K}, O_{\bar{K}}\right)}:=H^{0} L_{\left(\bar{K}, O_{\bar{K}}\right)}$ is an isomorphism. Therefore the canonical map $A_{d R}:=L \Omega_{\hat{O}_{\bar{K}}} \rightarrow L \Omega^{\cdot} \hat{(}_{\left.\bar{K}, O_{\bar{K}}\right)}$ is a filtered quasi-isomorphism.

Proof. For a finite extension $K^{\prime}$ of $K$ consider the log scheme $\operatorname{Spec}\left(K^{\prime}, O_{K^{\prime}}\right):=$ (Spec $K^{\prime}, \operatorname{Spec} O_{K^{\prime}}$ ). It is a log complete intersection over $O_{K}$ (see [O], 6.8). If $\pi$ is a generator of $O_{K^{\prime}} / O_{K}, f(t)$ its minimal polynomial, then, by [O1, 6.9], $\mathrm{L}_{\left(K^{\prime}, O_{K^{\prime}}\right)}$ is quasi-isomorphic to the cone of the multiplication by $f^{\prime}(\pi)$ map $O_{K^{\prime}} \rightarrow O_{K^{\prime}} \subset \mathfrak{m}_{K^{\prime}}^{-1}$. Thus $\mathrm{L}_{\left(K^{\prime}, O_{K^{\prime}}\right)}$ is acyclic in nonzero degrees, $\Omega_{\left(K^{\prime}, O_{K^{\prime}}\right)}:=H^{0} \mathrm{~L}_{\left(K^{\prime}, O_{K^{\prime}}\right)}$ is a cyclic $O_{K^{\prime}}$-module, and the canonical map $\Omega_{O_{K^{\prime}}} \rightarrow \Omega_{\left(K^{\prime}, O_{K^{\prime}}\right)}$ is an embedding with cokernel isomorphic to the residue field $O_{K^{\prime}} / \mathfrak{m}_{K^{\prime}}$. Now pass to the inductive limit, and use the fact that $\Omega_{O_{\bar{K}}}$ is $p$-divisible (see $\S 1.3$ ).

3.3. Consider the presheaf $(U, \bar{U}) \mapsto R \Gamma_{\mathrm{dR}}^{\natural}(U, \bar{U}):=R \Gamma\left(\bar{U}, L \Omega_{(\hat{U}, \bar{U})}\right)$ of filtered $\mathrm{E}_{\infty}$ $\operatorname{dg} O_{K}$-algebras on $\mathcal{V} a r_{K}^{\mathrm{ss}}$. Denote by $\mathcal{A}_{\mathrm{dR}}^{\natural}$ its h-sheafification (2.6.1); this is an h-sheaf of filtered $\mathrm{E}_{\infty} O_{K}$-algebras on $\mathcal{V} r_{\bar{K}}$ (as above, we see it as the projective system of quotients modulo $\left.F^{i}\right)$. Since $\mathrm{A}_{\mathrm{dR}}=\mathcal{A}_{\mathrm{dR}}^{\natural}(\operatorname{Spec} \bar{K})$ by Lemma in $\S 3.2$, $\mathrm{A}_{\mathrm{dR}}$, viewed as a constant filtered h-sheaf, maps into $\mathcal{A}_{\mathrm{dR}}^{\natural}$.

Theorem ( $p$-adic Poincaré lemma). The maps $A_{d R} \otimes^{L} \mathbb{Z} / p^{n} \rightarrow \mathcal{A}_{d R}^{\natural} \otimes^{L} \mathbb{Z} / p^{n}$ are filtered quasi-isomorphisms of h-sheaves on $\mathcal{V} a r_{\bar{K}}$.

For a proof, see $\S 4$. Assuming it, let us define the $p$-adic period map $\rho$. 
3.4. The Hodge-Deligne filtration. For this subsection, $K$ is any field of characteristic 0 . Consider the presheaf $(V, \bar{V}) \mapsto R \Gamma_{\mathrm{dR}}(V, \bar{V}):=R \Gamma\left(\bar{V}, \Omega_{(V, \bar{V})}\right)$ of filtered $\mathrm{E}_{\infty}$ dg $K$-algebras on $\mathcal{V} a r_{K}^{\text {nc }}$. Let $\mathcal{A}_{\mathrm{dR}}$ be its h-sheafification (2.6.1), which is an h-sheaf

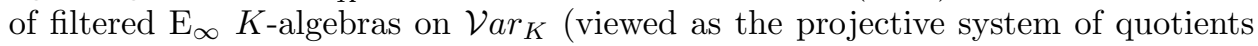
modulo $\left.F^{i}\right)$. For any $X \in \mathcal{V} a r_{K}$ set

$$
R \Gamma_{\mathrm{dR}}(X):=R \Gamma\left(X_{\mathrm{h}}, \mathcal{A}_{\mathrm{dR}}\right) .
$$

This is Deligne's de Rham complex of $X$ equipped with Deligne's Hodge filtration.

Proposition. (i) For $(V, \bar{V}) \in \mathcal{V} a r_{K}^{n c}$ the canonical map $R \Gamma_{d R}(V, \bar{V}) \rightarrow R \Gamma_{d R}(V)$ is a filtered quasi-isomorphism.

(ii) The differential of $R \Gamma_{d R}(X)$ is strictly compatible with the filtration. $H_{d R}^{i}(X):=$ $H^{i} R \Gamma_{d R}(X)$ are $K$-vector spaces of dimension equal to $\operatorname{dim} H_{e t}^{i}\left(X_{\bar{K}}, \mathbb{Q}_{p}\right)$.

(iii) For any smooth variety $X$ there is a canonical (nonfiltered) quasi-isomorphism $R \Gamma\left(X, \Omega_{X}\right) \stackrel{\sim}{\rightarrow} R \Gamma_{d R}(X)$.

Proof. By Lefschetz's principle, we can assume that $K=\mathbb{C}$. For $(V, \bar{V}) \in \mathcal{V} a r_{\mathbb{C}}^{\text {nc }}$ the maps $R \Gamma_{\mathrm{dR}}(V, \bar{V}) \rightarrow R \Gamma_{\mathrm{dR}}\left(V, \Omega_{V}\right) \rightarrow R \Gamma\left(V_{\mathrm{cl}}, \mathbb{C}\right)$ are quasi-isomorphisms by $\mathrm{Gr}$. Thus for any h-hypercovering $14(Y, \bar{Y}$. $) / X$ of $X$ in $\mathcal{V}_{K}^{\text {nc }}{ }_{K}$ the cohomological descent (see Remark in $\S 2.4$ ) yields a canonical quasi-isomorphism $R \Gamma\left(\bar{Y}_{\cdot}, \Omega_{\left(Y_{,}, \bar{Y}_{.}\right)}\right) \stackrel{\sim}{\rightarrow}$ $R \Gamma\left(X_{\mathrm{cl}}, \mathbb{C}\right)$. If we equip $R \Gamma\left(X_{\mathrm{cl}}, \mathbb{C}\right)$ with the Hodge-Deligne filtration of mixed Hodge theory $\mathrm{D}$, then this is a filtered quasi-isomorphism. Therefore we have a canonical filtered quasi-isomorphism $R \Gamma_{\mathrm{dR}}(X) \stackrel{\sim}{\rightarrow} R \Gamma\left(X_{\mathrm{cl}}, \mathbb{C}\right)$. Now (i) and the second assertion of (ii) are clear; the first assertion of (ii) follows from mixed Hodge theory. The quasi-isomorphism in (iii) is $R \Gamma\left(X, \Omega_{X}\right) \stackrel{\sim}{\rightarrow} R \Gamma\left(Y, \Omega_{Y .}\right) \tilde{\leftarrow}$ $R \Gamma\left(\bar{Y}_{.}, \Omega_{\left(Y_{,}, \bar{Y}_{.}\right)}\right)$, where the arrows are quasi-isomorphisms by the cohomological descent (since $R \Gamma\left(X, \Omega_{X}\right) \stackrel{\sim}{\rightarrow} R \Gamma\left(X_{\mathrm{cl}}, \mathbb{C}\right)$ ).

3.5. We return to the setting of $\S 3.3$, so $K$ is our $p$-adic field. Let $X$ be any variety over $\bar{K}$. It yields a filtered $\mathrm{E}_{\infty} O_{K}$-algebra

$$
R \Gamma_{\mathrm{dR}}^{\natural}(X):=R \Gamma\left(X_{\mathrm{h}}, \mathcal{A}_{\mathrm{dR}}^{\natural}\right) .
$$

Since $\mathrm{A}_{\mathrm{dR}} \otimes \mathbb{Q}=\bar{K}$ (see Remark (i) in $\S 1.5$ ), $R \Gamma_{\mathrm{dR}}^{\natural}(X) \otimes \mathbb{Q}$ is a $\bar{K}$-algebra. By Remark in $\S 3.2$, we have a filtered quasi-isomorphism of $\mathrm{E}_{\infty} \bar{K}$-algebras

$$
R \Gamma_{\mathrm{dR}}^{\natural}(X) \otimes \mathbb{Q} \stackrel{\sim}{\rightarrow} R \Gamma_{\mathrm{dR}}(X) .
$$

Let us compute $R \Gamma_{\mathrm{dR}}^{\natural}(X) \widehat{\otimes} \mathbb{Z}_{p}$. Consider the morphisms of filtered complexes $R \Gamma\left(X_{\text {ét }}, \mathbb{Z}\right) \otimes{ }^{L} \mathrm{~A}_{\mathrm{dR}} \stackrel{\sim}{\rightarrow} R \Gamma\left(X_{\text {ét }}, \mathrm{A}_{\mathrm{dR}}\right) \rightarrow R \Gamma\left(X_{\mathrm{h}}, \mathrm{A}_{\mathrm{dR}}\right) \rightarrow R \Gamma\left(X_{\mathrm{h}}, \mathcal{A}_{\mathrm{dR}}^{\natural}\right)=R \Gamma_{\mathrm{dR}}^{\natural}(X)$. After applying $\cdot \otimes^{L} \mathbb{Z} / p^{n}$, the arrows become filtered quasi-isomorphisms (the first one by Remark in $\S 2.4$, the second one by the Poincaré lemma in $\S 3.3)$, so we get a filtered quasi-isomorphism $R \Gamma\left(X_{\text {ét }}, \mathbb{Z} / p^{n}\right) \otimes^{L} \mathrm{~A}_{\mathrm{dR}} \stackrel{\sim}{\rightarrow} R \Gamma_{\mathrm{dR}}^{\natural}(X) \otimes^{L} \mathbb{Z} / p^{n}$. Since $R \Gamma_{\text {ét }}\left(X, \mathbb{Z}_{p}\right)=\operatorname{holim} R \Gamma\left(X_{\text {ét }}, \mathbb{Z} / p^{n}\right)=R \Gamma\left(X_{\text {ét }}, \mathbb{Z}\right) \widehat{\otimes} \mathbb{Z}_{p}$ (see $\left.\S 1.1\right)$ is a perfect $\mathbb{Z}_{p^{-}}$ complex and $R \Gamma\left(X_{\text {ét }}, \mathbb{Z} / p^{n}\right)=R \Gamma_{\text {ét }}\left(X, \mathbb{Z}_{p}\right) \otimes_{\mathbb{Z}_{p}}^{L} \mathbb{Z} / p^{n}$, one has, passing to the homotopy limit as in $\S 1.1, R \Gamma_{\text {ét }}\left(X, \mathbb{Z}_{p}\right) \otimes_{\mathbb{Z}_{p}}^{L}\left(\mathrm{~A}_{\mathrm{dR}} \widehat{\otimes} \mathbb{Z}_{p}\right) \stackrel{\sim}{\rightarrow} R \Gamma_{\mathrm{dR}}^{\natural}(X) \widehat{\otimes} \mathbb{Z}_{p}$. Tensoring by $\mathbb{Q}$, we get a filtered quasi-isomorphism of filtered $\mathrm{E}_{\infty} \mathrm{B}_{\mathrm{dR}}^{+}$-algebras (see (1.5.1))

$$
\beta: R \Gamma_{\text {ét }}\left(X, \mathbb{Q}_{p}\right) \otimes \mathrm{B}_{\mathrm{dR}}^{+} \stackrel{\sim}{\rightarrow} R \Gamma_{\mathrm{dR}}^{\natural}(X) \widehat{\otimes} \mathbb{Q}_{p} .
$$

\footnotetext{
${ }^{14}$ Here we view $X$ as an h-sheaf on $\mathcal{V}_{K} r_{K}^{\text {nc }}$, so $(Y ., \bar{Y}$. $)$ is a simplicial object of $\mathcal{V} a r_{\bar{K}}^{\text {nc }}$ equipped with an augmentation map $Y$. $\rightarrow X$ that makes $Y$. an h-hypercovering of $X$.
} 
Let $\alpha: R \Gamma_{\mathrm{dR}}(X) \otimes_{\bar{K}} \mathrm{~B}_{\mathrm{dR}}^{+} \rightarrow R \Gamma_{\mathrm{dR}}^{\natural}(X) \widehat{\otimes} \mathbb{Q}_{p}$ be the $\mathrm{B}_{\mathrm{dR}}^{+}$-linear extension of the composition $R \Gamma_{\mathrm{dR}}(X) \stackrel{\sim}{\rightarrow} R \Gamma_{\mathrm{dR}}^{\natural}(X) \otimes \mathbb{Q} \rightarrow R \Gamma_{\mathrm{dR}}^{\natural}(X) \widehat{\otimes} \mathbb{Q}_{p}$, where the first arrow is inverse to (3.5.2) and the second one comes from the canonical map ? $\rightarrow$ ? $\widehat{\otimes} \mathbb{Z}_{p}$. We get a morphism of filtered $\mathrm{E}_{\infty} \mathrm{B}_{\mathrm{dR}}^{+}$-algebras

$$
\rho=\rho_{\mathrm{dR}}:=\beta^{-1} \alpha: R \Gamma_{\mathrm{dR}}(X) \otimes_{\bar{K}} \mathrm{~B}_{\mathrm{dR}}^{+} \rightarrow R \Gamma_{\text {ét }}\left(X, \mathbb{Q}_{p}\right) \otimes_{\mathbb{Q}_{p}} \mathrm{~B}_{\mathrm{dR}}^{+} .
$$

Remarks. (i) The Galois group $\operatorname{Gal}(\bar{K} / K)$ acts on $\mathcal{V} r_{\bar{K} \mathrm{~h}}$ and on both sides of (3.5.4) by transport of structure, and $\rho^{+}$is evidently compatible with this action. In particular, if $X$ is defined over $K$, i.e., $X=X_{K} \otimes_{K} \bar{K}$, then $R \Gamma_{\mathrm{dR}}(X)=$ $R \Gamma_{\mathrm{dR}}\left(X_{K}\right) \otimes_{K} \bar{K}$, and we can rewrite (3.5.4) as a $\operatorname{Gal}(\bar{K} / K)$-equivariant morphism

$$
\rho: R \Gamma_{\mathrm{dR}}\left(X_{K}\right) \otimes_{K} \mathrm{~B}_{\mathrm{dR}}^{+} \rightarrow R \Gamma_{\text {ét }}\left(X, \mathbb{Q}_{p}\right) \otimes_{\mathbb{Q}_{p}} \mathrm{~B}_{\mathrm{dR}}^{+} .
$$

(ii) The map $\rho$ does not change if we replace $K$ by any of its finite extensions that are contained in $\bar{K}$ (see Remark in $\S 2.6$ ).

3.6. Theorem. The $B_{d R}$-linear extension of $\rho$ is a filtered quasi-isomorphism: for any $X \in \mathcal{V} r_{\bar{K}}$ one has

$$
\rho: R \Gamma_{d R}(X) \otimes_{\bar{K}} B_{d R} \stackrel{\sim}{\rightarrow} R \Gamma_{e ́ t}\left(X, \mathbb{Q}_{p}\right) \otimes_{\mathbb{Q}_{p}} B_{d R} .
$$

Proof. (a) The case of $X=\mathbb{G}_{m}=\mathbb{G}_{m \bar{K}}$ : The $\bar{K}$-line $H_{\mathrm{dR}}^{1}\left(\mathbb{G}_{m}\right)=\operatorname{gr}_{F}^{1} H_{\mathrm{dR}}^{1}\left(\mathbb{G}_{m}\right)$ is generated by $d \log t$. The $\mathbb{Z}_{p}$-line $H_{\text {ét }}^{1}\left(\mathbb{G}_{m}, \mathbb{Z}_{p}\right)(1)=H_{\text {ét }}^{1}\left(\mathbb{G}_{m}, \mathbb{Z}_{p}(1)\right)$ is generated by the class $\operatorname{cl}(\mathfrak{k})$ of the Kummer $\mathbb{Z}_{p}(1)$-torsor $\mathfrak{k}=\varliminf \mathfrak{k}_{n}, \mathfrak{k}_{n}:=\left(t^{1 / p^{n}}\right)$. Due to the canonical identification $\mathbb{C}_{p}(1) \stackrel{\sim}{\rightarrow} \mathfrak{m}_{\mathrm{dR}} / \mathfrak{m}_{\mathrm{dR}}^{2}=\operatorname{gr}_{F}^{1} \mathrm{~B}_{\mathrm{dR}}$, see $\S 1.4, \S 1.5$, we can view $c l(\mathfrak{k})$ as a generator of the $\mathbb{C}_{p}$-line $H_{\text {ét }}^{1}\left(\mathbb{G}_{m}, \mathbb{Q}_{p}\right) \otimes \operatorname{gr}_{F}^{1} \mathrm{~B}_{\mathrm{dR}}$.

Lemma. One has $g r_{F}^{1}(\rho)(d \log t)=\operatorname{cl}(\mathfrak{k}) \in H_{e ̂ t}^{1}\left(\mathbb{G}_{m}, \mathbb{Q}_{p}\right) \otimes g r_{F}^{1} B_{d R}$.

Proof of Lemma. We make a mod $p^{n}$ computation. Consider the ss-pair $\left(\mathbb{G}_{m}, \overline{\mathbb{G}}_{m}\right)$, $\overline{\mathbb{G}}_{m}:=\mathbb{P}_{O_{\bar{K}}}^{1}$. One has $\operatorname{gr}_{F}^{1} L \Omega_{\left(\hat{\mathbb{G}}_{m}, \overline{\mathbb{G}}_{m}\right)}=\Omega_{\left(\mathbb{G}_{m}, \overline{\mathbb{G}}_{m}\right)}^{1}[-1]$, so $d \log t \in \Gamma\left(\overline{\mathbb{G}}_{m}, \Omega_{\left(\mathbb{G}_{m}, \overline{\mathbb{G}}_{m}\right)}^{1}\right)$ is a 1 -cocycle in $\operatorname{gr}_{F}^{1} R \Gamma_{\mathrm{dR}}^{\natural}\left(\mathbb{G}_{m}\right)$. As in $\S 1.1$, set $C_{n}:=\mathcal{C}$ one $\left(p^{n}: \mathbb{Z} \rightarrow \mathbb{Z}\right)$. Let $d \log c l\left(\mathfrak{k}_{n}\right)$ be the image of the class $c l\left(\mathfrak{k}_{n}\right)$ of $\mathfrak{k}_{n}$ by the composition $H^{1}\left(\mathbb{G}_{m}\right.$ ét,$\left.\mu_{p^{n}}\right)$ $\rightarrow H^{1}\left(\mathbb{G}_{m}\right.$ ét, $\left.\operatorname{gr}_{F}^{1} \mathrm{~A}_{\mathrm{dR}} \otimes C_{n}\right) \rightarrow \operatorname{gr}_{F}^{1} R \Gamma_{\mathrm{dR}}^{\natural}\left(\mathbb{G}_{m}\right) \otimes C_{n}$, where the first arrow comes from the coefficient maps $\mu_{p^{n}} \stackrel{d \log }{\longrightarrow} \Omega_{O_{\bar{K}} p^{n}} \hookrightarrow \Omega_{O_{\bar{K}}}[-1] \otimes C_{n}=\operatorname{gr}_{F}^{1} \mathrm{~A}_{\mathrm{dR}} \otimes C_{n}$. To prove the lemma, we will show that the image of $d \log t$ by the embedding $\operatorname{gr}_{F}^{1} R \Gamma_{\mathrm{dR}}^{\natural}\left(\mathbb{G}_{m}\right) \hookrightarrow \operatorname{gr}_{F}^{1} R \Gamma_{\mathrm{dR}}^{\natural}\left(\mathbb{G}_{m}\right) \otimes C_{n}$ is homologous to $d \log c l\left(\mathfrak{k}_{n}\right)$.

Let $\mathbb{G}_{m}$ be a copy of $\mathbb{G}_{m}$ with parameter $\tilde{t}$, and $\pi: \mathbb{G}_{m} \rightarrow \mathbb{G}_{m}$ be the projection

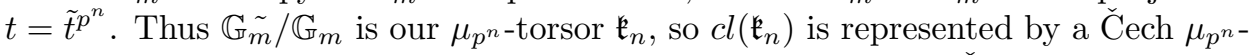
cocycle $c\left(\mathfrak{k}_{n}\right)$ for the étale covering $\mathbb{G}_{m} \tilde{m} / \mathbb{G}_{m}$. The corresponding Čech hypercovering is the twist of $\mathbb{G} \tilde{m}$ by the universal $\mu_{p^{n}}$-torsor $\mathfrak{t}_{n}$ over the classifying simplicial space $B_{\mu_{p^{n}}}$, so for any sheaf $\mathcal{F}$ the Cech complex of $\mathbb{G} \tilde{m} / \mathbb{G}_{m}$ with coefficients in $\mathcal{F}$ is the cochain complex $C^{\cdot}\left(\mu_{p^{n}}, \Gamma\left(\mathbb{G}_{m}, \mathcal{F}\right)\right)$ for $\mu_{p^{n}}$ acting on sections by the translations. The 1-cocycle $c\left(\mathfrak{k}_{n}\right)$ is the identity map $\mu_{p^{n}} \rightarrow \mu_{p^{n}}=\Gamma\left(\mathbb{G}_{m}, \mu_{p^{n}}\right)$.

Our $\pi$ extends to the h-covering of semi-stable pairs $\left(\mathbb{G}_{m}, \overline{\mathbb{G}}_{m}\right) \rightarrow\left(\mathbb{G}_{m}, \overline{\mathbb{G}}_{m}\right)$, and the Čech hypercovering extends to a hypercovering in $\mathcal{V} a r_{\bar{K}}^{\mathrm{ss}}$ which is the $\mathfrak{t}_{n^{-}}$ twist of $\left(\mathbb{G}_{m}, \overline{\mathbb{G}}_{m}\right)$. So one has a canonical map $C^{\cdot}\left(\mu_{p^{n}}, \Gamma\left(\overline{\mathbb{G}}_{m}, \Omega_{\left(\mathbb{G}_{m}, \overline{\mathbb{G}}_{m}\right)}^{1}\right)\right)[-1] \rightarrow$ $\operatorname{gr}_{F}^{1} R \Gamma_{\mathrm{dR}}^{\natural}\left(\mathbb{G}_{m}\right)$; hence $C^{\cdot}\left(\mu_{p^{n}}, \Gamma\left(\overline{\mathbb{G}}_{m}^{\tilde{m}}, \Omega_{\left(\mathbb{G}_{\tilde{m}}, \overline{\mathbb{G}}_{\tilde{m}}\right)}^{1}\right)\right)[-1] \otimes C_{n} \rightarrow \operatorname{gr}_{F}^{1} R \Gamma_{\mathrm{dR}}^{\natural}\left(\mathbb{G}_{m}\right) \otimes C_{n}$. Both $d \log t$ and $d \log c\left(\mathfrak{k}_{n}\right)$ are 1-cocycles in $C^{\cdot}\left(\mu_{p^{n}}, \Gamma\left(\overline{\mathbb{G}}_{m}^{\sim}, \Omega_{\left(\mathbb{G}_{\tilde{m}}, \overline{\mathbb{G}}_{\tilde{m}}\right)}^{1}\right)\right)[-1] \otimes C_{n}$ : namely, $d \log t \in C^{0}\left(\mu_{p^{n}}, \Gamma\left(\overline{\mathbb{G}}_{m}^{\sim}, \Omega_{\left(\mathbb{G}_{\tilde{m}}, \overline{\mathbb{G}}_{\tilde{m}}\right)}^{1}\right)[-1]\right)$ and $d \log c\left(\mathfrak{k}_{n}\right) \in C^{1}\left(\mu_{p^{n}}, \Omega_{O_{\bar{K}} p^{n}}\right)$ 
$\subset C^{1}\left(\mu_{p^{n}}, \Gamma\left(\overline{\mathbb{G}}_{m}, \Omega_{\left(\mathbb{G}_{m}, \overline{\mathbb{G}}_{m}\right)}^{1}\right)[-1] \otimes C_{n}\right)$. Their difference is the differential of the 0 cochain $d \log \tilde{t} \in C^{0}\left(\mu_{p^{n}}, \Gamma\left(\overline{\mathbb{G}}_{m}^{\sim}, \Omega_{(\mathbb{G} \tilde{m}, \overline{\mathbb{G}} \tilde{m})}^{1}\right)\right) \subset C^{0}\left(\mu_{p^{n}}, \Gamma\left(\overline{\mathbb{G}}_{m}, \Omega_{\left(\mathbb{G} \tilde{m}, \overline{\mathbb{G}}_{m}\right)}^{1}\right)[-1] \otimes C_{n}\right)$, q.e.d.

We see that for $X=\mathbb{G}_{m}$ the map $\rho$ of (3.6.1) is a filtered quasi-isomorphism. It provides a canonical generator $\rho(d \log t) / \operatorname{cl}(\mathfrak{k})$ of $\mathfrak{m}_{\mathrm{dR}}(-1)$. Thus we have a canonical identification between $H_{\text {ét }}\left(X, \mathbb{Q}_{p}(n)\right) \otimes \mathrm{B}_{\mathrm{dR}}$ and $H_{\dot{e} \mathrm{t}}\left(X, \mathbb{Q}_{p}\right) \otimes \mathrm{B}_{\mathrm{dR}}$ with the filtration shifted by $n$, etc.

Remark. The above generator is equal to Fontaine's generator from [F3, 1.5.4]. Indeed, they coincide modulo $\mathfrak{m}_{\mathrm{dR}}^{2}(-1)$ by the lemma, and both are $\operatorname{Gal}(\bar{K} / K)$ invariant (see Remark in §3.5). Since $H^{0}\left(\operatorname{Gal}(\bar{K} / K), \mathfrak{m}_{\mathrm{dR}}^{2}(-1)\right)=0$, we are done.

(b) Compatibility with the Gysin maps: Let $i: Y \hookrightarrow X$ be a closed codimension 1 embedding of smooth varieties. It yields the Gysin isomorphisms $i_{* \mathrm{dR}}: R \Gamma_{\mathrm{dR}}(Y) \stackrel{\sim}{\rightarrow}$ $R \Gamma_{\mathrm{dR} Y}(X)(1)[2]:=\mathcal{C}$ one $\left(R \Gamma_{\mathrm{dR}}(X) \rightarrow \Gamma_{\mathrm{dR}}(X \backslash Y)\right)(1)[1], i_{* \mathbb{Q}_{p}}: R \Gamma_{\text {ét }}\left(Y, \mathbb{Q}_{p}\right) \stackrel{\sim}{\rightarrow}$ $R \Gamma_{\text {ét } Y}\left(X, \mathbb{Q}_{p}\right)(1)[2]$. Let us show that $\rho$ commutes with the Gysin maps.

Consider the deformation to the normal cone diagram

$$
\begin{array}{ccccc}
\mathcal{L} & \hookrightarrow & X_{\mathbb{A}^{1}} & \hookleftarrow & X \\
\uparrow & & \uparrow & & \uparrow \\
Y & \hookrightarrow & Y_{\mathbb{A}^{1}} & \hookleftarrow & Y .
\end{array}
$$

Here $Y_{\mathbb{A}^{1}}=Y \times \mathbb{A}^{1}, X_{\mathbb{A}^{1}}$ is $X \times \mathbb{A}^{1}$ with $Y \times\{0\}$ blown up, the left arrow is the zero section of the normal bundle $\mathcal{L}$ to $Y$ in $X$, and the bottom embeddings are $y \mapsto(y, 0),(y, 1)$. It yields a commutative diagram of the de Rham cohomology

$$
\begin{array}{ccccc}
R \Gamma_{\mathrm{dR} Y}(\mathcal{L})(1)[2] & \leftarrow & R \Gamma_{\mathrm{dR} Y_{\mathbb{A}^{1}}\left(X_{\mathbb{A}^{1}}\right)(1)[2]} & \rightarrow & R \Gamma_{\mathrm{dR} Y}(X)(1)[2] \\
\uparrow & & \uparrow & & \uparrow \\
R \Gamma_{\mathrm{dR}}(Y) & \leftarrow & R \Gamma_{\mathrm{dR}}\left(Y_{\mathbb{A}^{1}}\right) & \rightarrow & R \Gamma_{\mathrm{dR}}(Y),
\end{array}
$$

where the vertical arrows are the Gysin isomorphisms and the horizontal ones are pullbacks. There is a similar diagram for the $\mathbb{Q}_{p}$-cohomology. The horizontal maps are filtered quasi-isomorphisms, so, since $\rho$ is compatible with pullbacks, we see that the Gysin compatibility for $Y \hookrightarrow X$ amounts to one for $Y \hookrightarrow \mathcal{L}$.

So we can assume that $X$ is a line bundle $\mathcal{L}$ over $Y$ and $i$ its zero section. Now the source of both $i_{*}$ 's are dg algebras, the targets are modules over them (due to the projection $\mathcal{L} \rightarrow Y$ ), and $i_{*}$ 's are morphisms of modules. Thus it suffices to check that $\rho$ identifies the images of 1 . The assertion is local with respect to $Y$; hence we can assume that $\mathcal{L}$ is trivial. By base change, we reduced to the case when $Y$ is a point, where we are done by (a).

(c) The case of a smooth projective $X$ : Let us check that the morphism of bigraded rings $\operatorname{gr}_{F} \rho^{*}: \operatorname{gr}_{F} H_{\mathrm{dR}}^{*}(X) \otimes_{\bar{K}} \operatorname{gr}_{F}^{*} \mathrm{~B}_{\mathrm{dR}} \rightarrow H_{\text {êt }}^{*}\left(X, \mathbb{Q}_{p}\right) \otimes_{\mathbb{Q}_{p}} \operatorname{gr}_{F} \mathrm{~B}_{\mathrm{dR}}$ is an isomorphism. It is an isomorphism for $*=0$. By (b), $\operatorname{gr}_{F}^{1} \rho^{2}$ identifies the classes $c$ of a hyperplane section. Since the product with $c^{\operatorname{dim} X}$ identifies $H^{0}$ and $H^{2 \operatorname{dim} X}$, $\operatorname{gr}_{F} \rho^{2 \operatorname{dim} X}$ is an isomorphism. Therefore, since $\operatorname{gr}_{F}^{*} \rho^{*}$ is compatible with the Poincaré pairing for classes of opposite degrees and the latter is nondegenerate, $\operatorname{gr}_{F}^{*} \rho^{*}$ is injective. Since $\operatorname{dim}_{\bar{K}} H_{\mathrm{dR}}^{*}(X)=\operatorname{dim}_{\mathbb{Q}_{p}} H_{\text {êt }}^{*}\left(X, \mathbb{Q}_{p}\right)$, we are done.

(d) The case of $X=\bar{X} \backslash D$, where $\bar{X}$ is smooth projective, $D$ is a strict normal crossings divisor: Let $Y$ be an irreducible component of $D, D^{\prime}$ be the union of the other components; set $X^{\prime}:=\bar{X} \backslash D^{\prime}, Y^{\prime}:=Y \backslash D^{\prime}$. By induction by the number 
of components $D$ (starting with (c)), we can assume that the theorem holds for $X^{\prime}$ and $Y^{\prime}$. By (b), $\rho$ provides a morphism between the exact Gysin triangles for $\left(Y^{\prime}, X^{\prime}\right)$. It is a filtered quasi-isomorphism on the $X^{\prime}$ and $Y^{\prime}$ terms; hence it is a filtered quasi-isomorphism on the $X$ term, q.e.d.

(e) The case of arbitrary $X$ : If $Y . / X$ is any h-hypercovering of $X$, then the canonical map $R \Gamma_{\mathrm{dR}}(X) \rightarrow R \Gamma_{\mathrm{dR}}(Y$.) (which is the total complex of the cosimplicial system of filtered complexes $\left.R \Gamma_{\mathrm{dR}}\left(Y_{i}\right)\right)$ is a filtered quasi-isomorphism by the construction of $R \Gamma_{\mathrm{dR}}$, and $R \Gamma_{\text {ét }}\left(X, \mathbb{Q}_{p}\right) \stackrel{\sim}{\rightarrow} R \Gamma_{\text {ét }}\left(Y, \mathbb{Q}_{p}\right)$ by cohomological descent (see Remark in 2.4). Thus if $\rho$ is a filtered quasi-isomorphism for every $Y_{i}$, then it is a filtered quasi-isomorphism for $X$. We are done, since, by de Jong (or Hironaka), one can find $Y . / X$ with $Y_{i}$ as in (d).

Remark. $\rho$ is compatible with Chern classes of vector bundles: Indeed, $c_{i}(E)$ are determined in the usual way by $c_{1}\left(\mathcal{O}(1)_{\mathbb{P}(E)}\right)$, so it suffices to show that $\rho$ identifies $c_{1}$ 's of line bundles. Notice that the construction of $\rho$ extends tautologically to simplicial schemes. By (a) above, $\rho$ identifies the de Rham and étale Chern classes of the universal line bundle over the classifying simplicial scheme $B_{\mathbb{G}_{m}}$. For a line bundle $\mathcal{L}$ on $X$, choose a finite open covering $\left\{U_{i}\right\}$ of $X$ such that $\mathcal{L}$ is trivial on $U_{i}$; let $\pi: X^{\sim} \rightarrow X$ be the Čech hypercovering. Since $\pi$ yields an isomorphism between the cohomology, it suffices to check that $\rho$ identifies the Chern classes of $\pi^{*} \mathcal{L}$. This is true since $\pi^{*} \mathcal{L}$ is the pullback of the universal line bundle by a map $X^{\sim} \rightarrow B_{\mathbb{G}_{m}}$.

\section{Proof of the Poincaré lemma}

4.1. Pick any $(V, \bar{V}) \in \mathcal{V} a r_{\bar{K}}^{\mathrm{ss}}$.

Proposition. One has $L_{(V, \bar{V})} \stackrel{\sim}{\rightarrow} \Omega_{(V, \bar{V})}$, the $\mathcal{O}_{\bar{V}}$-module $\Omega_{\langle V, \bar{V}\rangle}:=\Omega_{(V, \bar{V}) /\left(\bar{K}, O_{\bar{K}}\right)}$ is locally free of finite rank, and there is a canonical short exact sequence

$$
0 \rightarrow \mathcal{O}_{\bar{V}} \otimes_{O_{\bar{K}}} \Omega_{O_{\bar{K}}} \rightarrow \Omega_{(V, \bar{V})} \rightarrow \Omega_{\langle V, \bar{V}\rangle} \rightarrow 0 .
$$

Proof. We can assume that $V$ is connected, so $(V, \bar{V})$ is the base change of a semistable $K$-pair $(U, \bar{U})$ as in $2.2(\mathrm{c})$, i.e., $(V, \bar{V})=\left(U_{\bar{K}}, \bar{U}_{O_{\bar{K}}}\right)$. For any finite extension $K^{\prime}$ of $K_{U}$, consider an arithmetic $K$-pair $\left(U_{K^{\prime}}, \bar{U}_{O_{K^{\prime}}}\right):=\left(U \otimes_{K_{U}} K^{\prime}, \bar{U} \otimes_{O_{K_{U}}} O_{K^{\prime}}\right)$. Set $\Omega_{\langle U, \bar{U}\rangle}:=\Omega_{(U, \bar{U}) /\left(K_{U}, O_{K_{U}}\right)}, \Omega_{\left\langle U_{K^{\prime}}, \bar{U}_{O_{K^{\prime}}}\right\rangle}:=\Omega_{\left(U_{K^{\prime}}, \bar{U}_{O_{K^{\prime}}}\right) /\left(K^{\prime}, O_{K^{\prime}}\right)}$.

Lemma. The log scheme $\left(U_{K^{\prime}}, \bar{U}_{O_{K^{\prime}}}\right)$ coincides with the pullback of $(U, \bar{U})$ by the map Spec $\left(K^{\prime}, O_{K^{\prime}}\right) \rightarrow \operatorname{Spec}\left(K_{U}, O_{K_{U}}\right)$ in the category of log schemes.

Assume the lemma for a moment. The map $(U, \bar{U}) \rightarrow \operatorname{Spec}\left(O_{K_{U}}, K_{U}\right)$ is $\log$ smooth and integral; by the lemma, $\left(U_{K^{\prime}}, \bar{U}_{O_{K^{\prime}}}\right) \rightarrow \operatorname{Spec}\left(O_{K^{\prime}}, K^{\prime}\right)$ enjoys the same properties. So, by [Ol, 8.34], $\mathrm{L}_{\left(U_{K^{\prime}}, \bar{U}_{O_{K^{\prime}}}\right) /\left(K^{\prime}, O_{K^{\prime}}\right)} \stackrel{\sim}{\rightarrow} \Omega_{\left\langle U_{K^{\prime}}, \bar{U}_{O_{K^{\prime}}}\right\rangle}=O_{K^{\prime}} \otimes_{O_{K_{U}}}$ $\Omega_{\langle U, \bar{U}\rangle}$, which is a locally free $\mathcal{O}_{\bar{U}_{O_{K^{\prime}}}}$-module of finite rank. Since $\mathrm{L}_{\left(K^{\prime}, O_{K^{\prime}}\right)} \stackrel{\sim}{\rightarrow}$ $\Omega_{\left(K^{\prime}, O_{K^{\prime}}\right)}$ (see the proof of Lemma in $\left.\S 3.2\right)$, the canonical exact triangle $([\mathrm{Ol}, 8.18]$ ) $\mathcal{O}_{\bar{U}_{O_{K^{\prime}}}} \otimes_{O_{K^{\prime}}} \mathrm{L}_{\left(K^{\prime}, O_{K^{\prime}}\right)} \rightarrow \mathrm{L}_{\left(U_{K^{\prime}}, \bar{U}_{\left.O_{K^{\prime}}\right)}\right)} \rightarrow \mathrm{L}_{\left(U_{K^{\prime}}, \bar{U}_{O_{K^{\prime}}}\right) /\left(K^{\prime}, O_{K^{\prime}}\right)}$ reduces to the short exact sequence $0 \rightarrow \mathcal{O}_{\bar{U}_{O_{K^{\prime}}}} \otimes_{O_{K^{\prime}}} \Omega_{\left(K^{\prime}, O_{K^{\prime}}\right)} \rightarrow \Omega_{\left(U_{K^{\prime}}, \bar{U}_{O_{K^{\prime}}}\right)} \rightarrow \Omega_{\left\langle U_{K^{\prime}}, \bar{U}_{O_{K^{\prime}}}\right\rangle} \rightarrow 0$. Pass to the limit by all $K^{\prime} \subset \bar{K}$ and use the lemma in $\S 3.2$; we are done.

Proof of Lemma. The underlying scheme of the pullback log scheme is $\bar{U}_{O_{K^{\prime}}}$. Let us show that its $\log$ structure map $\mathcal{M} \rightarrow \mathcal{O}_{\bar{U}_{O_{K^{\prime}}}} \cap j_{*} \mathcal{O}_{U_{K^{\prime}}}^{\times}$is an isomorphism. The assertion is étale local, so we can assume that $\bar{U}$ is étale over $\operatorname{Spec} O_{K_{U}}\left[t_{a}, t_{b}, t_{c}\right] /$ 
$\left(\Pi t_{a}-\pi_{K_{U}}\right)$, where $a, b, c$ are in finite sets $A, B, C, \pi_{K_{U}}$ is a uniformizing parameter in $O_{K_{U}}$, and $U$ is the subscheme where all $t_{a}, t_{b}$ are invertible. The log structure of $(U, \bar{U})$ is fine with a chart $\mathbb{N}[A \sqcup B] \rightarrow \mathcal{O}_{\bar{U}}$, which sends generators $m_{a}, m_{b}$ of $\mathbb{N}[A \sqcup B]$ to $t_{a}, t_{b}$. Therefore $15 \bar{U}_{O_{K^{\prime}}}$ is étale over Spec $O_{K^{\prime}}\left[t_{a}, t_{b}, t_{c}\right] /\left(\Pi t_{a}-\pi_{K^{\prime}}^{e}\right)$, where $e$ is the ramification index of $K^{\prime} / K_{U}, \pi_{K^{\prime}}$ is a uniformizing parameter in $O_{K^{\prime}}$, and the $\log$ structure $\mathcal{M}$ has a chart $M_{A, B} \rightarrow \mathcal{O}_{\bar{U}_{O_{K^{\prime}}}}$, where $M_{A, B}$ is the quotient of $\mathbb{N}[A \sqcup B] \oplus \mathbb{N}$ modulo the relation $\Pi m_{a}=m_{\pi}^{e}\left(m_{\pi}\right.$ is the generator of the last summand $\mathbb{N}$ ), the chart is $m_{a}, m_{b}, m_{\pi} \mapsto t_{a}, t_{b}, \pi_{K^{\prime}}$. Consider an embedding $M_{A, B} \hookrightarrow M_{A, B}^{w}:=e^{-1} \mathbb{N}[A] \oplus \mathbb{N}[B], m_{a}, m_{b}, m_{\pi} \mapsto m_{a}, m_{b}, \Pi m_{a}^{1 / e}$. Its image is formed by those $\Pi m_{a}^{n_{a} / e} \Pi m_{b}^{n_{b}}, n_{a}, n_{b} \in \mathbb{N}$, such that $n_{a}-n_{a^{\prime}} \in e \mathbb{Z}$ for any $a, a^{\prime} \in A$; thus $M_{A, B}$ is saturated. Now the $\log$ scheme $\left(\bar{U}_{O_{K^{\prime}}}, \mathcal{M}\right)$ is evidently $\log$ regular in the sense of $[\mathrm{K} 2,2.1]$; hence $\mathcal{M} \stackrel{\sim}{\rightarrow} \mathcal{O}_{\bar{U}_{O_{K^{\prime}}}} \cap j_{*} \mathcal{O}_{U_{K^{\prime}}}^{\times}$by $[\mathrm{K} 2,11.6]$, q.e.d 16

The reference to $[\mathrm{K} 2$ can be replaced by the next explicit argument: It suffices to show that the map of sheaves $\mathcal{M} / \mathcal{O}_{\bar{U}_{O_{K^{\prime}}}^{\times}}^{\times} \rightarrow\left(\mathcal{O}_{\bar{U}_{O_{K^{\prime}}}} \cap j_{*} \mathcal{O}_{U_{K^{\prime}}}^{\times}\right) / \mathcal{O}_{\bar{U}_{O_{K^{\prime}}}}^{\times}$is an isomorphism. The r.h.s. is the sheaf $\mathcal{D}$ of effective Cartier divisors supported on $\bar{U}_{O_{K^{\prime}}} \backslash U_{K^{\prime}}$. Let $\mathcal{D}^{w} \supset \mathcal{D}$ be the sheaf of the corresponding effective Weil divisors. For $x \in \bar{U}_{O_{K^{\prime}}}$, the fiber $\left(\mathcal{M} / \mathcal{O}_{\bar{U}_{O_{K}}}^{\times}\right)_{x}$ is the quotient $M_{A_{x}, B_{x}}$ of $M_{A, B}$, where $A_{x} \subset$ $A, B_{x} \subset B$ consist of those $a, b$ such that $t_{a}, t_{b}$ vanish at $x$. The map $M_{A_{x}, B_{x}} \rightarrow \mathcal{D}_{x}$ extends to an isomorphism $M_{A_{x}, B_{x}}^{w} \stackrel{\sim}{\rightarrow} \mathcal{D}_{x}^{w}$, which identifies a generator $m_{a}^{1 / e}$ with the reduced divisor $D_{a}$ of $t_{a}, m_{b}$ with $D_{b}:=\operatorname{div}\left(t_{b}\right)$. Thus $M_{A_{x}, B_{x}} \hookrightarrow \mathcal{D}_{x}$. To show that $\hookrightarrow$ is an isomorphism, we need to check that if $D=\Sigma n_{a} D_{a}+\Sigma n_{b} D_{b}$ is a Cartier divisor at $x$, then $n_{a}-n_{a^{\prime}} \in e \mathbb{Z}$ for any $a, a^{\prime} \in A_{x}$. We can assume that $A=\left\{a, a^{\prime}\right\}$, $B=C=\emptyset$, so $\bar{U}_{O_{K^{\prime}}}$ is a semi-stable curve over $O_{K^{\prime}}$. The exceptional divisor of its minimal desingularization $\tilde{U}_{O_{K^{\prime}}}$ is a chain of $e-1$ projective lines $P_{1}, \ldots, P_{e-1}$ with self-intersection indices $\left(P_{i}, P_{i}\right)=-2$. Let $\tilde{D}=n_{a} D_{a}^{b}+n_{1} P_{1}+\ldots+n_{e-1} P_{e-1}+$ $n_{a^{\prime}} D_{a^{\prime}}^{b}$ be the pullback of $D$ to $\tilde{U}_{O_{K^{\prime}}}$; here $D_{a}^{b}, D_{a^{\prime}}^{b}$ are strict transforms of $D_{a}$, $D_{a^{\prime}}$. One has $\left(\tilde{D}, P_{i}\right)=0$, i.e., $n_{i-1}-2 n_{i}+n_{i+1}=0$ or $n_{i}-n_{i-1}=n_{i+1}-n_{i}$, where $n_{0}:=n_{a}, n_{e}:=n_{a^{\prime}}$. Thus $n_{a^{\prime}}-n_{a}=e\left(n_{1}-n_{a}\right) \in e \mathbb{Z}$, and we are done.

4.2. Set $\Omega_{\langle V, \bar{V}\rangle}^{a}:=\Lambda_{\mathcal{O}_{\bar{V}}}^{a} \Omega_{\langle V, \bar{V}\rangle}$. Consider (4.1.1) as a 2-step filtration on $\Omega_{(V, \bar{V})}$; it splits locally since $\Omega_{\langle V, \bar{V}\rangle}$ is locally free. Passing to derived exterior powers, we get for any $m$ a finite increasing filtration $I$. on $\operatorname{gr}_{F}^{m} L \Omega_{(\hat{V}, \bar{V})}^{*}=\left(L \Lambda_{\mathcal{O}_{\bar{V}}}^{m} \Omega_{(V, \bar{V})}\right)[-m]$ with $\operatorname{gr}_{a}^{I} \operatorname{gr}_{F}^{m} L \Omega_{(\hat{V}, \bar{V})}=\Omega_{\langle V, \bar{V}\rangle}^{a} \otimes_{O_{\bar{K}}} \operatorname{gr}_{F}^{m-a} \mathrm{~A}_{\mathrm{dR}}[-a]$, hence on $\operatorname{gr}_{F}^{m} R \Gamma_{\mathrm{dR}}^{\natural}(V, \bar{V})$ with

$$
\operatorname{gr}_{a}^{I} \operatorname{gr}_{F}^{m} R \Gamma_{\mathrm{dR}}^{\natural}(V, \bar{V})=R \Gamma\left(\bar{V}, \Omega_{\langle V, \bar{V}\rangle}^{a}\right) \otimes_{O_{\bar{K}}}^{L} \operatorname{gr}_{F}^{m-a} \mathrm{~A}_{\mathrm{dR}}[-a] .
$$

Let $\mathcal{G}^{a}$ be the h-sheafification (see (2.6.1)) of the complex of presheaves $(V, \bar{V}) \mapsto$ $R \Gamma\left(\bar{V}, \Omega_{\langle V, \bar{V}\rangle}^{a}\right)$ on $\mathcal{V} a r_{\bar{K}}^{\mathrm{ss}}$. This is a complex of h-sheaves of $O_{\bar{K}}$-modules on $\mathcal{V} a r_{\bar{K}}$. Its cohomology $H^{b} \mathcal{G}^{a}$ is h-sheafification of the presheaf $(V, \bar{V}) \mapsto H^{b}\left(\bar{V}, \Omega_{\langle V, \bar{V}\rangle}^{a}\right)$ on $\mathcal{V} a r_{\bar{K}}^{\mathrm{ss}}$. Our $I$. is a filtration on the presheaf $(V, \bar{V}) \mapsto \operatorname{gr}_{F}^{m} R \Gamma_{\mathrm{dR}}^{\natural}(V, \bar{V})$; passing to h-sheafification, we get a finite filtration $I$. on $\operatorname{gr}_{F}^{m} \mathcal{A}_{\mathrm{dR}}^{\natural}$ with span $[0, m]$ and

$$
\operatorname{gr}_{a}^{I} \operatorname{gr}_{F}^{m} \mathcal{A}_{\mathrm{dR}}^{\natural}=\mathcal{G}^{a} \otimes_{O_{\bar{K}}}^{L} \operatorname{gr}_{F}^{m-a} \mathrm{~A}_{\mathrm{dR}}[-a] .
$$

\footnotetext{
${ }^{15}$ We replace one $t_{a} \in \mathcal{O}\left(\bar{U}_{O_{K^{\prime}}}\right)$ by $t_{a} \pi_{K^{\prime}}^{e} / \pi_{K_{U}}$.

${ }^{16} \mathrm{I}$ am grateful to Luc Illusie for the proof.
} 
Notice that the bottom cohomology $H^{0}$ of the bottom term $I_{0}=\operatorname{gr}_{0}^{I}$ is the constant sheaf $O_{\bar{K}}$ and $\mathcal{C}$ one $\left(\operatorname{gr}_{F}^{m} \mathrm{~A}_{\mathrm{dR}} \rightarrow \operatorname{gr}_{F}^{m} \mathcal{A}_{\mathrm{dR}}^{\natural}\right)=\operatorname{gr}_{F}^{m} \mathcal{A}_{\mathrm{dR}}^{\natural} / H^{0} I_{0}$. Therefore, by (4.2.2), the Poincaré lemma follows from the next assertion:

Theorem. The cohomology $H^{b} \mathcal{G}^{a}$ are $h$-sheaves of $\mathbb{Q}$ - (hence $\bar{K}$-) vector spaces for $(a, b) \neq(0,0)$.

Remark. The $p$-divisiblity of $H^{b} \mathcal{G}^{0}, b \neq 0$, was first proved by Bhatt [Bh1, 8.0.1].

Exercise. Consider a presheaf $(V, \bar{V}) \mapsto R \Gamma\left(\bar{V}, L \Omega_{(\hat{V}, \bar{V}) /\left(\bar{K}, O_{\bar{K}}\right)}\right)$; let $\mathcal{A}_{\mathrm{dR}}^{\text {naive }}$ be its h-sheafification. One has an evident map $\mathcal{C}$ one $\left(F^{1} \mathrm{~A}_{\mathrm{dR}} \rightarrow \mathcal{A}_{\mathrm{dR}}^{\natural}\right) \rightarrow \mathcal{A}_{\mathrm{dR}}^{\text {naive }}$. Show that the theorem implies that it is a filtered quasi-isomorphism; i.e., the triangle $F^{1} \mathrm{~A}_{\mathrm{dR}} \rightarrow \mathcal{A}_{\mathrm{dR}}^{\natural} \rightarrow \mathcal{A}_{\mathrm{dR}}^{\text {naive }}$ is exact in the filtered derived category of h-sheaves.

4.3. We deduce the above theorem from a more concrete assertion. As in $\S 4.1$, for an ss-pair $(U, \bar{U})$ over $K$ we have the locally free $\mathcal{O}_{\bar{U}}$-module of $\log$ differentials $\Omega_{\langle U, \bar{U}\rangle}:=\Omega_{(U, \bar{U}) /\left(K_{U}, O_{K_{U}}\right)}$ and its exterior powers $\Omega_{\langle U, \bar{U}\rangle}^{a}:=\Lambda^{a} \Omega_{\langle U, \bar{U}\rangle}$.

Let $f:\left(U^{\prime}, \bar{U}^{\prime}\right) \rightarrow(U, \bar{U})$ be a map in $\mathcal{V} a r_{K}^{\mathrm{ss}}$ or $\mathcal{V} a r_{K}^{\mathrm{ss}}$. We say that $f$ is (Hodge) $p$-negligible if the morphisms $\left(\tau_{>0} R \Gamma\left(\bar{U}, \mathcal{O}_{\bar{U}}\right)\right) \otimes^{L} \mathbb{Z} / p \rightarrow\left(\tau_{>0} R \Gamma\left(\bar{U}^{\prime}, \mathcal{O}_{\bar{U}^{\prime}}\right)\right) \otimes^{L} \mathbb{Z} / p$ and $R \Gamma\left(\bar{U}, \Omega_{\langle U, \bar{U}\rangle}^{a}\right) \otimes^{L} \mathbb{Z} / p \rightarrow R \Gamma\left(\bar{U}^{\prime}, \Omega_{\left\langle U^{\prime}, \bar{U}^{\prime}\right\rangle}^{a}\right) \otimes^{L} \mathbb{Z} / p, a>0$, in $D^{b}\left(O_{K_{U}} / p\right)$, resp. $D^{b}\left(O_{\bar{K}} / p\right)$, vanish.

Remark. For $(U, \bar{U}) \in \mathcal{V} a r_{K}^{\text {ss }}$ and a point $K_{U} \rightarrow \bar{K}$, one has $R \Gamma\left(\bar{U}_{O_{\bar{K}}}, \Omega_{\left\langle U_{\bar{K}}, \bar{U}_{\bar{K}_{\bar{K}}}\right\rangle}^{a}\right)=$ $R \Gamma\left(\bar{U}, \Omega_{\langle U, \bar{U}\rangle}^{a}\right) \otimes_{O_{K}}^{L} O_{\bar{K}}$. Therefore the base change functor ${\mathcal{V} a r_{K}^{\mathrm{ss}}}^{\mathrm{s}} \rightarrow \mathcal{V}^{\mathrm{s}} r_{\bar{K}}^{\mathrm{ss}}$ (see $(2.2 .2))$ preserves $p$-negligible maps.

Theorem. Every $U \in \mathcal{V}$ ar ${ }_{K}^{\text {ss }}$ admits a p-negligible $h$-covering. Ditto for $\bar{K}$-pairs.

The theorem implies the one in $\S 4.2$ : Indeed, the $\bar{K}$-assertion shows that one has $\left(\tau_{>0} \mathcal{G}^{0}\right) \otimes^{L} \mathbb{Z} / p=0$ and $\mathcal{G}^{a} \otimes^{L} \mathbb{Z} / p=0$ for $a>0$; since for a complex $\mathcal{G}$ the multiplication by $p$ on $H \cdot \mathcal{G}$ is invertible if and only if $\mathcal{G} \otimes^{L} \mathbb{Z} / p=0$, we are done. Thus it yields the Poincaré lemma.

The above remark shows that the $K$-version of the theorem implies the $\bar{K}$-one. The proof of the $K$-version takes the rest of the section.

4.4. For the rest of §4, "pair" means "arithmetic K-pair" (see §2.2). We need further input from de Jong. A morphism $f:(C, \bar{C}) \rightarrow(S, \bar{S})$ of pairs is said to be a family of pointed curves (over $(S, \bar{S})$ ) if the map $\bar{C}_{S}:=f^{-1}(S) \rightarrow S$ is smooth of relative dimension 1 with irreducible geometric fibers, and $D_{f S}:=\bar{C}_{S} \backslash C$, viewed as a reduced scheme, is étale over $S$. Such an $f$ is semi-stable if, in addition, $\bar{C} / \bar{S}$ is a semi-stable family of curves, and the closure $D_{f}$ of $D_{f S}$ in $\bar{C}$ (the horizontal divisor), viewed as a reduced scheme, is étale over $\bar{S}$ and intersects each fiber of $f$ at smooth points. A section $e:(S, \bar{S}) \rightarrow(C, \bar{C})$ of $f$ is said to be nice if $e(\bar{S})$ intersects fibers of $f$ at smooth points and $D_{f} \cap e(\bar{S})=\emptyset$. Families of pointed curves over $(S, \bar{S})$ form a category $\mathcal{C}_{(S, \bar{S})}$ in the obvious manner, and a morphism of bases $\psi:\left(S^{\prime}, \bar{S}^{\prime}\right) \rightarrow(S, \bar{S})$ yields an evident pullback functor $\mathcal{C}_{(S, \bar{S})} \rightarrow \mathcal{C}_{\left(S^{\prime}, \bar{S}^{\prime}\right)}$ which preserves semi-stable families. A morphism $f^{\prime} \rightarrow f$ in $\mathcal{C}_{(S, \bar{S})}$ is called an alteration if $\left(C^{\prime}, \bar{C}^{\prime}\right)$ is an alteration of $(C, \bar{C})$; it is a semi-stable alteration (of $f$ ) if, in addition, $f^{\prime}$ is semi-stable.

Theorem. (a) Any family $f:(C, \bar{C}) \rightarrow(S, \bar{S})$ of pointed curves with $f: \bar{C} \rightarrow \bar{S}$ projective admits a semi-stable alteration $f^{\prime}$ h-locally over $(S, \bar{S})$. 
(b) One can find $f^{\prime}$ as above which has a nice section e. Moreover, for a given closed subscheme $P \subset \bar{C}$ such that $f(P)=\bar{S}$ and $P \cap \bar{C}_{S} \subset C$, one can find e such that the map $\bar{C}^{\prime} \rightarrow \bar{C}$ sends e $(\bar{S})$ to $P$.

(c) For any semi-stable family of pointed curves $f:(C, \bar{C}) \rightarrow(S, \bar{S})$ with $(S, \bar{S})$ a strict ss-pair, there exists a semi-stable alteration $m:(C, \tilde{C}) \rightarrow(C, \bar{C})$ of $f$ with $\left.m\right|_{C}=i d_{C}$ such that $m: \tilde{C} \rightarrow \bar{C}$ is an isomorphism over smooth points of $f$ and $(C, \tilde{C})$ is an ss-pair.

Proof. (c) is dJ1, 3.6]. (a) follows from [dJ2, 2.4 (i),(ii)] except that de Jong does not care to control the domain of smoothness of the semi-stable alteration of $f$. A miniscule modification of his argument permits us to do this; see Appendix 1. Alternatively, (a) follows directly from a far more precise result of Temkin [T, 1.5] 17

Let us check (b). Every pair has a canonical alteration by the union of normalizations of its irreducible components, so we assume all the way that $\bar{S}$ is normal and irreducible. Since $P$ as in (b) exists h-locally on $(S, \bar{S}) 18$ we can assume it is given. Replacing $(S, \bar{S})$ by its alteration $\left(P_{S}, P\right)$, we get a section $e$ of $f$ with image in $P$. Set $C^{b}:=C \backslash e(S)$. Then $\left(C^{b}, \bar{C}\right) \rightarrow(S, \bar{S})$ is a family of pointed curves; let $f^{\prime \prime}:\left(C^{\prime b}, \bar{C}^{\prime}\right) \rightarrow(S, \bar{S})$ be its semi-stable alteration as in (a). Let $D_{e}$ be the closure in $\bar{C}^{\prime}$ of the preimage $D_{e S}$ of $e(S)$. Then $D_{e}$ is an étale covering of $\bar{S} 19$ Let $C^{\prime}$ be the preimage of $C \subset \bar{C}$ in $\bar{C}^{\prime}$; then $\left(C^{\prime}, \bar{C}^{\prime}\right) \rightarrow(S, \bar{S})$ is a semi-stable alteration of $(C, \bar{C}) \rightarrow(S, \bar{S})$. Replacing $(S, \bar{S})$ by its alteration $\left(D_{e S}, D_{e}\right)$, we get a nice section of $\left(C^{\prime}, \bar{C}^{\prime}\right)$ which sits over $e$, hence over $P$.

Remark. In (c), every nice section of $(C, \bar{C})$ lifts to a nice section of $(C, \tilde{C})$.

Corollary. Any pair $(U, \bar{U})$ has an h-covering by ss-pairs $(C, \bar{C}), \operatorname{dim} C=\operatorname{dim} U$, for which there is a semi-stable family of pointed curves $f:(C, \bar{C}) \rightarrow(S, \bar{S})$ with a nice section such that $(S, \bar{S})$ is an ss-pair and $C$ is affine over $S$ (i.e., $f\left(D_{f}\right)=\bar{S}$ ).

Proof. It suffices to find an h-covering of $(U, \bar{U})$ by pairs $(C, \bar{C})$ with $\operatorname{dim} C=\operatorname{dim} U$ for which there exists a family of pointed curves $f:(C, \bar{C}) \rightarrow(S, \bar{S})$ with $C$ affine over $S$ and projective $\bar{S}, \bar{C}$. The theorem transforms it then, with an input from Remark (i) in $\S 2.5$ to preserve the dimension and de Jong's theorem in $\S 2.3$ to alter $(S, \bar{S})$ from (b) into a strict ss-pair, into a datum with all promised properties.

By de Jong's theorem in $\S 2.3$, we can assume that $(U, \bar{U})$ is an ss-pair and $\bar{U}$ is projective and irreducible 20 set $d=\operatorname{dim} U$. Pick any closed point $u \in U$. It suffices to find an open neighborhood $U^{\prime} \subset U$ of $U$, an alteration $(C, \bar{C})$ of $\left(U^{\prime}, \bar{U}\right)$, and a family of curves $f:(C, \bar{C}) \rightarrow(S, \bar{S})$ such that $f\left(D_{f}\right)=\bar{S}$.

Embed $\bar{U}$ into a projective space $\mathbb{P}_{O_{K}}^{N}$. By Bertini, there is a plane $H \subset \mathbb{P}^{N}$ defined over $K$ of codimension $d$ such that $u \notin H, H$ intersects $\bar{U}_{K}$ transversally, $H \cap \bar{U}_{K} \subset U$, and the codimension $d-1$ plane which contains $H$ and $u$ is transversal to $\bar{U}_{K}$ and $\bar{U}_{K} \backslash U$. Let $m: \bar{C} \rightarrow \bar{U}$ be the blowup at $\bar{U} \cap H_{O_{K}}, p: \bar{C} \rightarrow \mathbb{P}_{O_{K}}^{d-1}$ be the projection defined by $H$, and $\bar{C} \stackrel{f}{\rightarrow} \bar{S} \rightarrow \mathbb{P}_{O_{K}}^{d-1}$ be the Stein factorization of $p$ (so $\left.\bar{S}=\operatorname{Spec} p_{*} \mathcal{O}_{\bar{C}}\right)$. Let $D \subset \bar{C}$ be the union of $m^{-1}(\bar{U} \backslash U)$ and the exceptional divisor (viewed as a reduced scheme), and $S \subset \bar{S}_{K}$ be the maximal open subset

\footnotetext{
${ }^{17}$ To bring our datum to Temkin's setting, one flattens $\bar{C} / \bar{S}$ and $D_{f} / \bar{S}$ using [RG 5.2.2] and replaces $\bar{S}$ by its normalization.

${ }^{18}$ Indeed, one can cover $S$ by open subsets $\left\{S_{\alpha}\right\}$ such that $P$ exists for $\left(C_{S_{\alpha}}, \bar{C}\right) \rightarrow\left(S_{\alpha}, \bar{S}\right)$.

${ }^{19}$ Since $D_{f^{\prime b}}$ is étale over $\bar{S}, D_{e S}$ is open and closed in $D_{f^{\prime b} S}, f^{\prime b}\left(D_{e S}\right)=S$, and $\bar{S}$ is normal.

${ }^{20}$ We only need that $\bar{U}$ is projective and normal, and that $U$ is smooth.
} 
over which $f$ is smooth and $\left.f\right|_{D}$ is étale. Set $C:=f^{-1}(S) \backslash D$ and $U^{\prime}:=m(C)$; notice that $\left.m\right|_{C}: C \stackrel{\sim}{\rightarrow} U^{\prime}$. Then $U^{\prime},(C, \bar{C}), f$ satisfy the promised properties (one has $f\left(D_{f}\right)=\bar{S}$ since $D_{f}$ contains the exceptional divisor), q.e.d.

4.5. Let us return to the proof of the theorem in $\S 4.3$. We use induction by $\operatorname{dim} U$. By the corollary in $\S 4.4$, we can replace $(U, \bar{U})$ by $(C, \bar{C})$ as in loc. cit., so we have $f:(C, \bar{C}) \rightarrow(S, \bar{S})$ with a nice section $e$ and $C$ affine over $S$. Notice that $(C, \bar{C})$ is $\log$ smooth over $(S, \bar{S})$ and the line bundle $\omega_{f}:=\Omega_{(C, \bar{C}) /(S, \bar{S})}$ equals $f^{!}\left(\mathcal{O}_{\bar{S}}\right)[-1] \otimes \mathcal{O}_{\bar{C}}\left(D_{f}\right)$.

Key lemma. h-locally over $(S, \bar{S})$, one can find a semi-stable alteration $\phi: f^{\prime} \rightarrow f$ together with a nice section $e^{\prime}$ that lifts e such that $\left(C^{\prime}, \bar{C}^{\prime}\right)$ is an ss-pair and the pullback maps $\phi^{*}: R^{1} f_{*} \mathcal{O}_{\bar{C}} \rightarrow R^{1} f_{*}^{\prime} \mathcal{O}_{\bar{C}^{\prime}}, f_{*} \omega_{f} \rightarrow f_{*}^{\prime} \omega_{f^{\prime}}$ are divisible by $p 21$

For a proof, see $\S 4.6$. Assuming it for the moment, let us finish the proof of the theorem in $\S 4.3$. By Remark (i) in $\S 2.5$, we can assume that the h-localization of $(S, \bar{S})$ in the Key Lemma does not change $\operatorname{dim} S$. We will show that for some h-covering $\left(S^{\prime}, \bar{S}^{\prime}\right)$ of $(S, \bar{S})$ the composition $\left(C^{\prime}, \bar{C}^{\prime}\right)_{\left(S^{\prime}, \bar{S}^{\prime}\right)} \rightarrow\left(C^{\prime}, \bar{C}^{\prime}\right) \stackrel{\phi}{\longrightarrow}(C, \bar{C})$ is p-negligible.

For any $a$ consider the exact sequence

$$
0 \rightarrow f^{*} \Omega_{\langle S, \bar{S}\rangle}^{a} \rightarrow \Omega_{\langle C, \bar{C}\rangle}^{a} \rightarrow\left(f^{*} \Omega_{\langle S, \bar{S}\rangle}^{a-1}\right) \otimes \omega_{f} \rightarrow 0 .
$$

The section $e$ splits off $\Omega_{\langle S, \bar{S}\rangle}^{a} \hookrightarrow R f_{*} \Omega_{\langle C, \bar{C}\rangle}^{a}$ as a direct summand whose complement is $\mathcal{C}$ one $\left(\partial_{C}\right)$, where $\partial_{C}: \Omega_{\langle S, \bar{S}\rangle}^{a-1} \otimes f_{*} \omega_{f} \rightarrow \Omega_{\langle S, \bar{S}\rangle}^{a} \otimes R^{1} f_{*} \mathcal{O}_{\bar{C}}$ is the boundary map for (4.5.1) (one has $R^{1} f_{*} \omega_{f}=0$ since $f\left(D_{f}\right)=\bar{S}$ ). There is a similar splitting in case of $f^{\prime}$ provided by $e^{\prime}$, and the map $\phi^{*}: R f_{*} \Omega_{\langle C, \bar{C}\rangle}^{a} \rightarrow R f_{*}^{\prime} \Omega_{\left\langle C^{\prime}, \bar{C}^{\prime}\right\rangle}^{a}$ is compatible with the direct sum decompositions. Now $\phi^{*}$ is divisible by $p$ on the second summand: Indeed, the Key Lemma asserts that the morphism of two-term complexes $\phi^{*}$ : $\mathcal{C}$ one $\left(\partial_{C}\right) \rightarrow \mathcal{C}$ one $\left(\partial_{C^{\prime}}\right)$ is divisible by $p$ on each term; since these are morphisms of vector bundles on $O_{K}$-flat $\bar{S}$, our $p^{-1} \phi^{*}$ is uniquely defined and commutes with the differentials. Thus the map $\phi^{*} \otimes \operatorname{id}_{C_{1}}: \mathcal{C}$ one $\left(\partial_{C}\right) \otimes C_{1} \rightarrow \mathcal{C}$ one $\left(\partial_{C^{\prime}}\right) \otimes C_{1}$, where $C_{1}:=\mathcal{C}$ one $(p: \mathbb{Z} \rightarrow \mathbb{Z})$, is homotopic to zero. Apply $R \Gamma(\bar{S}, \cdot)$ and use the induction assumption to treat the first summand $R \Gamma\left(\bar{S}, \Omega_{\langle S, \bar{S}\rangle}^{a}\right)$; we are done.

4.6. Proof of Key Lemma. Consider the relative Picard $\bar{S}$-schemes $J:=\operatorname{Pic} c^{0}(\bar{C} / \bar{S})$ and $J^{b}:=\operatorname{Pic}^{0}\left(\left(\bar{C}, D_{f}\right) / \bar{S}\right)$ : the first scheme parametrizes line bundles $\mathcal{L}$ on $\bar{C}$ such that the restriction of $\mathcal{L}$ to the normalization of each irreducible component of any geometric fiber of $f$ has degree 0 ; the second one parametrizes pairs $(\mathcal{L}, \gamma)$, where $\mathcal{L}$ is as above and $\gamma$ is a trivialization of $\left.\mathcal{L}\right|_{D_{f}}$. Since $\left(\bar{C}, D_{f}\right)$ is a semi-stable $\bar{S}$-family of $d$-pointed curves, $d:=\operatorname{deg}\left(D_{f}\right)$, our $J$ and $J^{\mathrm{b}}$ are semi-abelian schemes (see $[\mathrm{R}]$ ), and $J^{\mathrm{b}}$ is an extension of $J$ by a torus $\mathbb{G}_{m}^{D_{f}} / \mathbb{G}_{m}$.

Over $S$ our $J^{b}$ is a generalized Jacobian; let $i: C \rightarrow J_{S}^{b}$ be the Abel-Jacobi map $i: C \rightarrow J_{S}^{\mathrm{b}}, x \mapsto \mathcal{O}_{\bar{C}}(x-e)$. Let $C^{\sim} \rightarrow C$ be the $i$-pullback of the multiplication by $p$ isogeny $p_{J^{b}}: J^{b} \rightarrow J^{b}$, and $\bar{C}^{\sim} \rightarrow \bar{C}$ be the normalization of $\bar{C}$ in $C^{\sim}$. Then $f^{\sim}:\left(C^{\sim}, \bar{C}^{\sim}\right) \rightarrow(S, \bar{S})$ is a family of pointed curves, which is an alteration of $f$. By the theorem in $\S 4.4$, h-locally over $(S, \bar{S})$ there is a semi-stable alteration $f^{\prime}$ of $f^{\sim}$

${ }^{21}$ As elements of the groups $\operatorname{Hom}_{\mathcal{O}_{\bar{S}}}\left(R^{1} f_{*} \mathcal{O}_{\bar{C}}, R^{1} f_{*}^{\prime} \mathcal{O}_{\bar{C}^{\prime}}\right), \operatorname{Hom}_{\mathcal{O}_{\bar{S}}}\left(f_{*} \omega_{f}, f_{*}^{\prime} \omega_{f^{\prime}}\right)$. 
with $\left(C^{\prime}, \bar{C}^{\prime}\right)$ semi-stable and equipped with a nice section $e^{\prime}$ which lies over $e$. Let us check that the alteration $\phi: f^{\prime} \rightarrow f$ satisfies the conditions of the Key Lemma.

Set $J^{\prime}:=P i c^{0}\left(\bar{C}^{\prime} / \bar{S}\right)$ and $J^{\prime \prime}:=P i c^{0}\left(\left(\bar{C}^{\prime}, D_{f}\right) / \bar{S}\right)$. We have the pullback morphisms $\phi^{*}: J \rightarrow J^{\prime}, J^{b} \rightarrow J^{\prime b}$ of our semi-abelian schemes; over $S$ we have the norm maps $\phi_{* S}: J_{S}^{\prime} \rightarrow J_{S}, J_{S}^{\prime b} \rightarrow J_{S}^{b}$. Both are compatible with the projections $J^{b} \rightarrow J, J^{\prime b} \rightarrow J^{\prime}$.

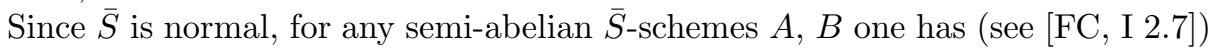

$$
\operatorname{Hom}(A, B) \stackrel{\sim}{\rightarrow} \operatorname{Hom}\left(A_{S}, B_{S}\right) .
$$

Thus $\phi_{* S}$ extends to morphisms $\phi_{*}: J^{\prime} \rightarrow J, J^{\prime b} \rightarrow J^{b}$.

Notice that $R^{1} f_{*} \mathcal{O}_{\bar{C}}$ is the Lie algebra of $J$, and, by Serre duality, $f_{*} \omega_{f}$ is dual to the Lie algebra of $J^{b}$; the same is true for $f^{\prime}$. Our $\phi^{*}: R^{1} f_{*} \mathcal{O}_{\bar{C}} \rightarrow R^{1} f_{*}^{\prime} \mathcal{O}_{\bar{C}^{\prime}}$ is the Lie algebra map for $\phi^{*}: J \rightarrow J^{\prime}$, and $\phi^{*}: f_{*} \omega_{f} \rightarrow f_{*}^{\prime} \omega_{f^{\prime}}$ is the map between the duals to the Lie algebras for $\phi_{*}: J^{\prime b} \rightarrow J^{b}$ (this is true over $S$, hence everywhere since $\bar{S}$ is $O_{K}$-flat).

By construction, $\phi_{* S}: J_{S}^{\prime b} \rightarrow J_{S}^{b}$ factors through $p_{J^{b}}$ over $S$; i.e., it is divisible by $p$ in $\operatorname{Hom}\left(J_{S}^{\prime b}, J_{S}^{\mathrm{b}}\right)$. By (4.6.1), $\phi_{*}$ is divisible by $p$ in $\operatorname{Hom}\left(J^{\prime b}, J^{\mathrm{b}}\right)$. Passing to Lie algebras, we see that $\phi^{*}: f_{*} \omega_{f} \rightarrow f_{*}^{\prime} \omega_{f^{\prime}}$ is divisible by $p$. Similarly, $\phi_{* S}: J_{S}^{\prime} \rightarrow J_{S}$ is divisible by $p$. Notice that $J_{S}, J_{S}^{\prime}$, being Jacobians of smooth projective curves, are self-dual abelian schemes, and $\phi_{S}^{*}: J_{S} \rightarrow J_{S}^{\prime}$ is dual to $\phi_{* S}$. Hence $\phi_{S}^{*}$ is divisible by $p$. So, by (4.6.1), $\phi^{*}: J \rightarrow J^{\prime}$ is divisible by $p$. Passing to Lie algebras, we see that $\phi^{*}: R^{1} f_{*} \mathcal{O}_{\bar{C}} \rightarrow R^{1} f_{*}^{\prime} \mathcal{O}_{\bar{C}^{\prime}}$ is divisible by $p$, q.e.d.

\section{APPENDIX}

Below is a proof of part (a) in the theorem from $\S 4.4$. It follows closely de Jong's argument from $\S \S 2-3$ of dJ2 with a minor change of the lemma below; we refer the reader to sections of dJ2 for details.

(i) (dJ2, 2.10]) One can assume that $\bar{S}$ is irreducible. By [RG, 5.2.2], there is a canonical modification of $\bar{S}$, which is projective and is an isomorphism over $S$, such that the strict transforms of $\bar{C}$ and $D_{f}$ are flat over $\bar{S}$. Passing to them, we can assume that all fibers of $f$ have dimension 1 , of $\left.f\right|_{D_{f}}$ have dimension 0 .

(ii) ( $\mathrm{dJ} 2,3.4-3.5])$ We say that a family of pointed curves is good if irreducible components of all its geometric fibers are curves whose normalization has genus $\geq 2$. A good alteration is an alteration with a good source.

Lemma. $f$ admits a good alteration h-locally over $(S, \bar{S})$.

Proof of Lemma. It suffices to find for any closed point $s$ in $S$ its open neighborhood $S_{(s)} \subset S$ and an alteration $\left(S_{(s)}^{\prime}, \bar{S}^{\prime}\right)$ of $\left(S_{(s)}, \bar{S}\right)$ such that the pullback of $f$ to $\left(S_{(s)}^{\prime}, \bar{S}^{\prime}\right)$ admits a good alteration. To do this, we define by induction a strictly increasing sequence of open subsets $\emptyset=V_{0} \subset V_{1} \subset \ldots$ of $\bar{S}$ and a sequence of finite extensions $F=F_{0} \subset F_{1} \subset \ldots$ of the field $F$ of rational functions on $\bar{C}$ such that the normalization $\bar{C}_{i}$ of $\bar{C}$ in $F_{i}$ has the following properties: (a) the map $\bar{C}_{i} \rightarrow \bar{S}$ is smooth at $s$, (b) the map $\pi_{i}: \bar{C}_{i} \rightarrow \bar{C}$ is étale at $D_{f s}$, (c) the normalizations of irreducible components of geometric fibers of $\bar{C}_{i}$ over points of $V_{i}$ have genus $\geq 2$. There is an open neighborhood $U_{i} \subset S$ of $s$ over which $\bar{C}_{i}$ is smooth and $\pi_{i}$ is étale at $D_{f}$. The induction stops when $V_{n}=\bar{S}$; set $S_{(s)}=U_{n}$. The promised good alteration is $\left(\pi_{n}^{-1}(C), \bar{C}_{n}\right)$ fibered over the normalization $\left(S_{(s)}^{\prime}, \bar{S}^{\prime}\right)$ of $\left(S_{(s)}, \bar{S}\right)$ in $F_{n}$. 
Let $x$ be the closed point of the closure of $s$ in $\bar{S}$. The induction produces simultaneously an auxiliary sequence of finite subsets $T_{0} \subset T_{1} \subset \ldots$ of closed points of $\bar{C}_{x}$; it starts with $T_{0}:=$ the union of $D_{f x}$ and the set of nonregular points of $\bar{C}_{x}$. The induction step: suppose we have $V_{i-1}, F_{i-1}, T_{i-1}$; let us construct $V_{i}$, $F_{i}, T_{i}$ assuming that $V_{i-1} \neq \bar{S}$. Let $y$ be any closed point in $\bar{S} \backslash V_{i-1}$. Since $\bar{S}$ is projective, there is an affine open $V$ which contains $x$ and $y$. Let $\mathcal{L}$ be a very ample line bundle on $\bar{C}$. Replacing it by a sufficiently high power, we can assume that $\Gamma\left(\bar{C}_{V}, \mathcal{L}\right) \rightarrow \Gamma\left(\bar{C}_{x}, \mathcal{L}\right) \times \Gamma\left(\bar{C}_{y}, \mathcal{L}\right)$. One can find a finite unramified extension 22 of $K$ with residue field $k^{\prime}$ and two sections $\gamma_{1}, \gamma_{2} \in \Gamma\left(\bar{C}_{V}, \mathcal{L}\right) \otimes_{O_{K}} O_{K^{\prime}}$ which do not vanish at the generic points of irreducible components of $\bar{C}_{x}, \bar{C}_{y}$, such that $t=\gamma_{1} / \gamma_{2}$ yields generically étale finite maps $t_{x}: \bar{C}_{x} \otimes k^{\prime} \rightarrow \mathbb{P}_{x}^{1} \otimes k^{\prime}, t_{y}: \bar{C}_{y} \otimes k^{\prime} \rightarrow \mathbb{P}_{y}^{1} \otimes k^{\prime}$ étale over $\{0,1, \infty\}$ and such that $t_{x}\left(T_{i-1}\right) \cap\{0,1, \infty\}=\emptyset$. Pick $\ell \geq 5$ prime to $p$, and let $F_{i}$ be an extension of $F_{i-1}$ generated by $K^{\prime}, \mu_{\ell}, t^{1 / \ell}$, and $(1-t)^{1 / \ell}$. Let $T_{i}$ be the union of $T_{i-1}$ and the set of ramification points of $t_{x}$. The normalization $\bar{C}_{i}$ of $\bar{C}$ in $F_{i}$ satisfies (a), (b), and satisfies (c) over some open set $V_{i}$ which contains $V_{i-1}$ and $y$. We are done.

(iii) It remains to show that every good $f$ admits a semi-stable alteration after a possible alteration of the base. The genus of the generic fiber of $f$ is $\geq 2$, so $\left(\bar{C}_{S}, D_{f S}\right)$ is a stable $n$-pointed curve over $S$ (where $n$ is the degree of $D_{f S}$ over $S)$. The Deligne-Mumford stack of stable $n$-pointed curves is proper, so, after replacing $(S, \bar{S})$ by an alteration, we can assume that $\left(\bar{C}_{S}, D_{f S}\right)$ extends to a stable $n$-pointed curve $\left(\bar{C}^{\prime}, D_{f^{\prime}}\right)$ over $\bar{S}$ (see [dJ2, 3.8]). We have a semi-stable family of pointed curves $f^{\prime}:\left(C^{\prime}, \bar{C}^{\prime}\right) \rightarrow(S, \bar{S}), C^{\prime}:=\bar{C}^{\prime} \backslash D_{f^{\prime}}$. By [dJ2, 3.10], the goodness of $f$ implies that, after a possible alteration of $\bar{S}$, the evident morphism $\bar{C}_{S}^{\prime} \rightarrow \bar{C}_{S}$ extends to a morphism $f^{\prime} \rightarrow f$, and we are done.

\section{ACKNOWLEDGMENTS}

This work was prompted by a discussion with Kobi Kremnizer (and watching the moon over a meadow in Oxford). The author is very grateful to him and to Bhargav Bhatt, Volodya Drinfeld, John Francis, Luc Illusie, David Kazhdan, Jacob Lurie, Madhav Nori, and Misha Temkin for explanations, questions, and corrections of mistakes.

\section{REFERENCES}

[B] A. Beilinson, On the crystalline period map, math. AG 1111.3316 (2011).

[Ber] L. Berger, Représentations p-adiques et équations différentielles, Inv. Math. 148 (2) (2002), 219-284. MR1906150 (2004a:14022)

[Bh1] B. Bhatt, Derived direct summands, Thesis, Princeton University (2010). MR2753219

[Bh2] B. Bhatt, p-adic derived de Rham cohomology, 2012.

[Col] P. Colmez, Les nombres algébriques sont denses dans $B_{d R}^{+}$, Périodes $p$-adiques, Astérisque 223, Soc. Math. France, 1994, pp. 103-111.

[Con] B. Conrad, Cohomological descent, http://math.stanford.edu/ ${ }^{\sim}$ conrad/.

[D] P. Deligne, Théorie de Hodge III, Publ. Math. IHES 44 (1974), 5-77. MR0498552 $(58: 16653 \mathrm{~b})$

[dJ1] A. J. de Jong, Smoothness, semi-stability and alterations, Publ. Math. IHES 83 (1996), 51-93. MR 1423020 (98e:14011)

[dJ2] A. J. de Jong, Families of curves and alterations, Ann. Inst. Fourier 47 (2) (1997), 599-621. MR:1450427 (98f:14019)

\footnotetext{
${ }^{22}$ If the residue field of $K$ is infinite, one can take $K^{\prime}=K$.
} 
[Fa1] G. Faltings, p-adic Hodge theory, J. Amer. Math. Soc. 1 (1988), 255-288. MR.924705 (89g:14008)

[Fa2] G. Faltings, Almost étale extensions, Cohomologies p-adiques et applications arithmétiques (II), Astérisque 279, Soc. Math. France, 2002, pp. 185-270. MR.1922831 (2003m:14031)

[FC] G. Faltings, C.-L. Chai, Degeneration of abelian varieties, Springer-Verlag, 1990. MR.1083353 (92d:14036)

[Far] L. Fargues, Letter to L. Illusie, 2010.

[F1] J.-M. Fontaine, Sur certains types de représentations p-adiques du groupe de Galois d'un corps local; construction d'un anneau de Barsotti-Tate, Ann. of Math. (2) 115 (1982), 529-577. MR657238 (84d:14010)

[F2] J.-M. Fontaine, Formes différentielles et modules de Tate des variétés abéliennes sur les corps locaux, Inv. Math. 65 (1982), 379-409. MR643559 (83k:14023)

[F3] J.-M. Fontaine, Le corps de périodes p-adiques, Périodes p-adiques, Astérisque 223, Soc. Math. France, 1994, pp. 59-101. MR.1293971 (95k:11086)

[F4] J.-M. Fontaine, Représentations p-adiques semi-stables, Périodes p-adiques, Astérisque 223, Soc. Math. France, 1994, pp. 113-184. MR.1293972 (95g:14024)

[Gr] A. Grothendieck, On the de Rham cohomology of algebraic varieties, Publ. Math. IHES 29 (1966), 95-103. MR0199194 (33:7343)

[GV] A. Grothendieck, J.-L. Verdier, Conditions de finitude. Topos et sites fibrés. Applications aux questions de passage à la limite, Théorie des topos et cohomologie étale de schémas (SGA 4), Tome 2, Lect. Notes in Math. 270, Springer-Verlag, 1972, pp. 163-340. MR0354653(50:7131)

[HS] V. Hinich, V. Schechtman, On homotopy limit of homotopy algebras, K-theory, Arithmetic and Geometry, Yu. I. Manin (Ed.), Lect. Notes in Math. 1289, Springer-Verlag, 1987, pp. 240-264. MR923138 (89d:55052)

[Ill1] L. Illusie, Complexe Cotangent et Déformations I, Lect. Notes in Math. 239, SpringerVerlag, 1971. MR0491680 (58:10886a)

[Ill2] L. Illusie, Complexe Cotangent et Déformations II, Lect. Notes in Math. 283, SpringerVerlag, 1972. MR0491681 (58:10886b)

$[\mathrm{J}] \quad$ U. Jannsen, On the $\ell$-adic cohomology of varieties over number fields and its Galois cohomology, Galois groups over $\mathbb{Q}$, Y. Ihara, K. Ribet, J.-P. Serre (Eds.), MSRI Publ. 16, Springer-Verlag, 1989, pp. 315-360. MR.1012170 (90i:11064)

[K1] K. Kato, Logarithmic structures of Fontaine-Illusie, Algebraic Analysis, Geometry, and Number Theory, The Johns Hopkins University Press, 1988, pp. 191-224. MR.1463703 (99b:14020)

[K2] K. Kato, Toric singularities, American Jour. of Math. 116 (5) (1994), 1073-1099. MR.1296725 (95g:14056)

[N1] W. Niziol, Semistable conjecture via K-theory, Duke Math. J. 141 (1) (2008), 151-178. MR.2372150 (2008m:14035)

[N2] W. Niziol, p-adic motivic cohomology in arithmetic, International Congress of Mathematicians, vol. II (2006), 459-472. MR2275605 (2007k:14029)

[N3] W. Niziol, On uniqueness of p-adic period morphisms, Pure Appl. Math. Q. 5 (1) (2009), 163-212. MR2520458(2011c:14058)

[Ol] M. Olsson, The logarithmic cotangent complex, Math. Ann. 333 (2005), 859-931. MR2195148 (2006j:14017)

[R] M. Raynaud, Spécialisation du foncteur de Picard, Publ. Math. IHES 38 (1970), 27-76. MR0282993 (44:227)

[RG] M. Raynaud, L. Gruson, Critères de platitude et de projectivité. Téchniques de "platification" d'un module, Inv. Math. 13 (1971), 1-89. MR0308104 (46:7219)

[SD] B. Saint-Donat, Téchniques de déscente cohomologique, Théorie des topos et cohomologie étale de schémas (SGA 4), Tome 2, Lect. Notes in Math. 270, Springer-Verlag, 1972, pp. 83162. MR 0354653(50:7131)

[SV] A. Suslin, V. Voevodsky, Singular homology of abstract algebraic varieties, Inv. Math. 123 (1996), 61-94. MR1376246 (97e:14030)

[T] M. Temkin, Stable modification of relative curves, J. Algebraic Geom. 19 (4) (2010), 603677. MR 2669727 (2011j:14064)

[Ts1] T. Tsuji, p-adic étale cohomology and crystalline cohomology in the semi-stable reduction case, Inv. Math. 137 (1999), 233-411. MR.1705837 (2000m:14024) 
[Ts2] T. Tsuji, Semi-stable conjecture of Fontaine-Jannsen: a survey, Cohomologies p-adiques et applications arithmetiques (II), Astérisque 279, Soc. Math. France, 2002, pp. 323-370. MR1922833 (2003g:14028)

[V1] J.-L. Verdier, Topologies et faisceaux, Théorie des topos et cohomologie étale de schémas (SGA 4), Tome 1, Lect. Notes in Math. 269, Springer-Verlag, 1972, pp. 219-264.

[V2] J.-L. Verdier, Fonctorialité de catégories de faisceaux, Théorie des topos et cohomologie étale de schémas (SGA 4), Tome 1, Lect. Notes in Math. 269, Springer-Verlag, 1972, pp. 265-298.

[V3] J.-L. Verdier, Cohomologie dans les topos, Théorie des topos et cohomologie étale de schémas (SGA 4), Tome 2, Lect. Notes in Math. 270, Springer-Verlag, 1972, pp. 1-82.

[Y] G. Yamashita, Théorie de Hodge p-adique pour les variétés ouvertes, C. R. A. S. 349 (21-22) (2011), 1127-1130.

Department of Mathematics, University of Chicago, Chicago, Illinois 60637

E-mail address: sasha@math.uchicago.edu 